Supporting Information

\title{
Amakusamine from a Psammocinia sp. Sponge: Isolation, Synthesis, and SAR Study on the Inhibition of RANKL-Induced Formation of Multinuclear Osteoclasts
}

Yuka Maeyama, ${ }^{\dagger}$ Yuta Nakashima, ${ }^{\dagger}$ Hikaru Kato, ${ }^{\dagger}$ Yuki Hitora,${ }^{\dagger}$ Kazuhiko Maki, ${ }^{\dagger}$ Natsumi Inada, ${ }^{\dagger}$ Shunya Murakami, ${ }^{\dagger}$ Tomoaki Inazumi, ${ }^{\S}$ Yuji Ise, ${ }^{\perp}$ Yukihiko Sugimoto, ${ }^{\S}$ Hayato Ishikawa, ${ }^{\sharp}$ and Sachiko Tsukamoto ${ }^{\dagger, *}$

${ }^{\dagger}$ Department of Natural Medicines, Graduate School of Pharmaceutical Sciences, Kumamoto University, Kumamoto 862-0973, Japan

$¥$ Graduate School of Science and Technology, Kumamoto University, Kumamoto 860-8555, Japan

$\S$ Department of Pharmaceutical Biochemistry, Graduate School of Pharmaceutical Sciences, Kumamoto University, Kumamoto 862-0973, Japan

${ }^{\perp}$ Sesoko Station, University of the Ryukyus, 3422 Sesoko, Motobu, Okinawa 905-0227, Japan

\# Graduate School of Pharmaceutical Sciences, Chiba University, Chiba 260-8675, Japan

*Corresponding Author

E-mail address: sachiko@kumamoto-u.ac.jp (S. Tsukamoto).

\section{Table of Contents}

Page S3 A picture of a Psammocinia sp. Sponge

Pages S3-S14 General Remarks for Chemical Synthesis. Synthesis of 2,3-Dibromo Compound 3. Synthesis of Amakusamine Derivatives 7-20 for SAR Study.

Page S15 Figure S1. ${ }^{1} \mathrm{H}$ NMR spectrum of $\mathbf{1}$.

Figure S2. ${ }^{13} \mathrm{C}$ NMR spectrum of $\mathbf{1}$.

Page S16 Figure S3. COSY spectrum of $\mathbf{1}$.

Figure S4. HSQC spectrum of $\mathbf{1}$.

Page S17 Figure S5. HMBC spectrum of 1.

Page S18 Figure S6. ${ }^{1} \mathrm{H}$ NMR spectra of synthetic (upper) and natural (lower) 1.

Page S19 Figure S7. ${ }^{13} \mathrm{C}$ NMR spectra of synthetic (upper) and natural (lower) 1.

Page S20 Figure S8. ${ }^{1} \mathrm{H}$ and ${ }^{13} \mathrm{C}$ NMR spectra of $\mathbf{3}$. 
Page S21 Figure S9. ${ }^{1} \mathrm{H}$ and ${ }^{13} \mathrm{C}$ NMR spectra of 7.

Page S22 Figure S10. ${ }^{1} \mathrm{H}$ and ${ }^{13} \mathrm{C}$ NMR spectra of $\mathbf{S 1}$.

Page S23 Figure S11. ${ }^{1} \mathrm{H}$ and ${ }^{13} \mathrm{C}$ NMR spectra of $\mathbf{S 2}$.

Page S24 Figure S12. ${ }^{1} \mathrm{H}$ and ${ }^{13} \mathrm{C}$ NMR spectra of 9.

Page S25 Figure S13. ${ }^{1} \mathrm{H}$ and ${ }^{13} \mathrm{C}$ NMR spectra of $\mathbf{1 0}$.

Page S26 Figure S14. ${ }^{1} \mathrm{H}$ and ${ }^{13} \mathrm{C}$ NMR spectra of $\mathbf{S 3}$.

Page S27 Figure S15. ${ }^{1} \mathrm{H}$ and ${ }^{13} \mathrm{C}$ NMR spectra of $\mathbf{1 1 .}$

Page S28 Figure S16. ${ }^{1} \mathrm{H}$ and ${ }^{13} \mathrm{C}$ NMR spectra of $\mathbf{1 2}$.

Page S29 Figure S17. ${ }^{1} \mathrm{H}$ and ${ }^{13} \mathrm{C}$ NMR spectra of 13.

Page S30 Figure S18. ${ }^{1} \mathrm{H}$ and ${ }^{13} \mathrm{C}$ NMR spectra of $\mathbf{1 4}$.

Page S31 Figure S19. ${ }^{1} \mathrm{H}$ and ${ }^{13} \mathrm{C}$ NMR spectra of $\mathbf{1 5}$.

Page S32 Figure S20. ${ }^{1} \mathrm{H}$ and ${ }^{13} \mathrm{C}$ NMR spectra of $\mathbf{1 6}$.

Page S33 Figure S21. ${ }^{1} \mathrm{H}$ and ${ }^{13} \mathrm{C}$ NMR spectra of 17.

Page S34 Figure S22. ${ }^{1} \mathrm{H}$ and ${ }^{13} \mathrm{C}$ NMR spectra of $\mathbf{1 8}$.

Page S35 Figure S23. ${ }^{1} \mathrm{H}$ and ${ }^{13} \mathrm{C}$ NMR spectra of $\mathbf{1 9}$.

Page S36 Figure S24. ${ }^{1} \mathrm{H}$ and ${ }^{13} \mathrm{C}$ NMR spectra of $\mathbf{2 0}$.

Page S37 Table S1. Calculated ${ }^{13} \mathrm{C}$ NMR data based on $\omega$ B97X-D/6-31G* for models $\boldsymbol{a}$ and $\boldsymbol{b}$ and comparisons with experimental data of $\mathbf{1}$. 


\section{A Picture of a Psammocinia sp. Sponge}

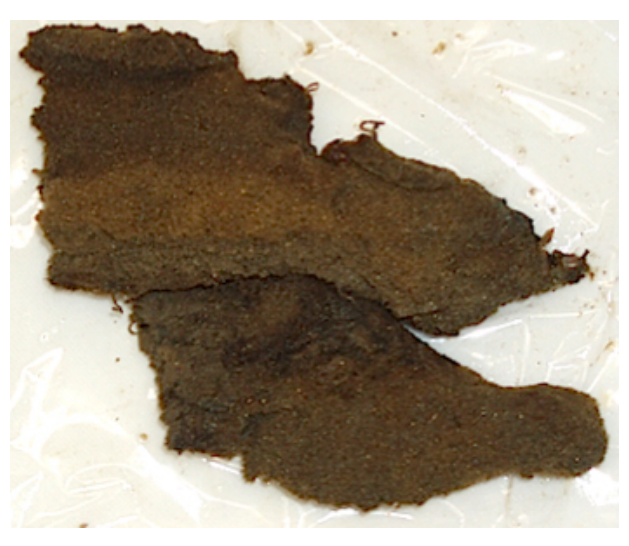

\section{General Remarks for Chemical Synthesis.}

All reactions were monitored by thin-layer chromatography using Merck 60 F254 precoated silica gel plates (0.25 mm thickness). FT-IR spectra were recorded on a SHIMADZU IR Affinity-IS and JASCO FT/IR-230 spectrophotometer. ${ }^{1} \mathrm{H}$ and ${ }^{13} \mathrm{C}$ NMR spectra were recorded on a JEOL ECX 500 FT-NMR spectrometer (500 MHz for ${ }^{1} \mathrm{H}$ NMR, $125 \mathrm{MHz}$ for ${ }^{13} \mathrm{C}$ NMR) and JNM ECZ-600 (600 MHz for ${ }^{1} \mathrm{H}$ $\mathrm{NMR}, 150 \mathrm{MHz}$ for $\left.{ }^{13} \mathrm{C} \mathrm{NMR}\right)$ instrument. Data for ${ }^{1} \mathrm{H}$ NMR are reported as chemical shift ( $\delta$ in ppm), multiplicity $(\mathrm{s}=$ singlet, $\mathrm{d}=$ doublet, $\mathrm{t}=$ triplet, $\mathrm{dd}=$ doubledoublet, $\mathrm{tt}=$ tripletriplet, $\mathrm{ddd}=$ doubledoubledoublet, $\mathrm{dt}=$ doubletriplet, $\mathrm{q}=$ quartet, quint $=$ quintet, $\mathrm{sext}=$ sextet, $\mathrm{m}=$ multiplet, $\mathrm{br}=$ broad), coupling constant (Hz), integration, and assignment. Data for ${ }^{13} \mathrm{C}$ NMR are reported as chemical shift. The high-resolution mass spectra were recorded on a BRUKER impact II and a JEOL AccuTOF LC-plus JMS-T100LP. Preparative thin layer chromatography was performed using Merck 60 F254 precoated silica gel plates $(0.25 \mathrm{~mm}$ thickness). Flash chromatography was performed using silica gel 60N (Kanto Chemical Co., Inc., Tokyo, Japan) and amino silica gel ( $\left.\mathrm{SiO}_{2}-\mathrm{NH}\right)$ (Fuji Silysia Co., Inc., Kasugai, Japan).

\section{Synthesis of 2,3-Bibromo Compound 3.}<smiles>c1cc2cc3c(cc2[nH]1)OCO3</smiles>

2

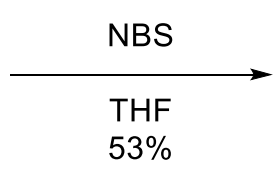

To a solution of 5,6-methylenedioxyindole (2, $20.0 \mathrm{mg}, 0.12 \mathrm{mmol})$ in THF $(330 \mu \mathrm{L}), N$ bromosuccinimide ( $49 \mathrm{mg}, 0.27 \mathrm{mmol}$ ) was slowly added at $0{ }^{\circ} \mathrm{C}$ under $\mathrm{Ar}$ atmosphere. The reaction mixture was stirred for $2 \mathrm{~h}$ at rt. The resulting mixture was quenched with saturated aqueous $\mathrm{NaHCO}_{3}$ 
solution. The aqueous layer was extracted three times with $\mathrm{CHCl}_{3}$. The combined organic layer was dried over $\mathrm{MgSO}_{4}$, and concentrated under reduced pressure. Flash chromatography $\left(\mathrm{SiO}_{2}, 50 \%\right.$ benzene $/ n$-hexane) provided 2,3-dibromo compound 3 (20.8 $\mathrm{mg}, 53 \%)$ as white crystals.

\section{6,7-Dibromo-5H-[1,3]dioxolo[4,5-f]indole (3).}

White crystals; mp $120-122^{\circ} \mathrm{C}$; IR (neat) $v_{\max } 3368,1461,1310,1167,1093,1034,938,829 \mathrm{~cm}^{-1}$; ${ }^{1} \mathrm{H}$ NMR $\left(500 \mathrm{MHz}, \mathrm{CDCl}_{3}\right) \delta 8.16$ (br. s, 1H), 6.87 (s, 1H), 6.76 (s, 1H), 5.96 (s, 2H); ${ }^{13} \mathrm{C}$ NMR $(125$ $\left.\mathrm{MHz}, \mathrm{CDCl}_{3}\right) \delta 146.1,144.2,130.6,122.0,106.6,101.1,98.0,94.5,91.9 ;$ HRESIMS $m / z 317.8746$ $[\mathrm{M}+\mathrm{H}]^{+}$(calcd for $\mathrm{C}_{9} \mathrm{H}_{6}{ }^{79} \mathrm{Br}_{2} \mathrm{NO}_{2}, 317.8760$ ).

\section{Synthesis of Amakusamine Derivatives 7-20 for SAR Study.}

Reduction of $\Delta^{2}$ double bond of amakusamine (1).<smiles>Brc1c2c(c(Br)c3[nH]ccc13)OCO2</smiles>

1

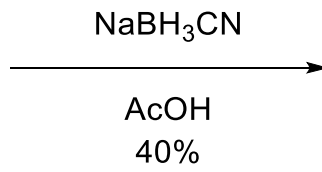

$40 \%$<smiles>Brc1c2c(c(Br)c3c1OCO3)NCC2</smiles>

7

To a solution of amakusamine $(1,10 \mathrm{mg}, 0.03 \mathrm{mmol})$ in acetic acid (AcOH, $400 \mu \mathrm{L})$, sodium cyanoborohydride $(5.7 \mathrm{mg}, 0.09 \mathrm{mmol})$ was added at $\mathrm{rt}$ under Ar atmosphere. The reaction mixture was stirred for $23 \mathrm{~h}$ at rt. The resulting mixture was quenched with saturated aqueous $\mathrm{NaHCO}_{3}$ solution at $0{ }^{\circ} \mathrm{C}$. The aqueous layer was extracted three times with $\mathrm{CHCl}_{3}$. The combined organic layer was dried over $\mathrm{MgSO}_{4}$, and concentrated under reduced pressure. The crude materials were purified by flash chromatography $\left(\mathrm{SiO}_{2}, 25 \% \mathrm{CHCl}_{3} / n\right.$-hexane) to provide $7(4.0 \mathrm{mg}, 40 \%)$ as white crystals.

\section{4,8-Dibromo-6,7-dihydro-5H-[1,3]dioxolo[4,5-f]indole (7).}

White crystals; mp $157-160{ }^{\circ} \mathrm{C}$; IR (neat) $v_{\max } 3343,2903,2834,1275,1076,1032,934 \mathrm{~cm}^{-1} ;{ }^{1} \mathrm{H}$ NMR (500 MHz, $\left.\mathrm{CDCl}_{3}\right) \delta 6.00$ (s, 2H), 3.85 (br. s, 1H), 3.64 (t, $\left.J=8.7 \mathrm{~Hz}, 2 \mathrm{H}\right), 3.08$ (t, $J=8.7 \mathrm{~Hz}$, $2 \mathrm{H}) ;{ }^{13} \mathrm{C}$ NMR $\left(125 \mathrm{MHz}, \mathrm{CDCl}_{3}\right) \delta 145.5,145.0,138.4,121.7,101.6,98.6,84.8,47.2,31.9$; HRESIMS $m / z 319.8933[\mathrm{M}+\mathrm{H}]^{+}$(calcd for $\mathrm{C}_{9} \mathrm{H}_{8}{ }^{79} \mathrm{Br}_{2} \mathrm{NO}_{2}, 319.8916$ ).

Compound $\mathbf{8}$ was prepared by reported protocol. ${ }^{1}$ 
<smiles>O=Cc1cc2c(cc1[N+](=O)[O-])OCO2</smiles>

4

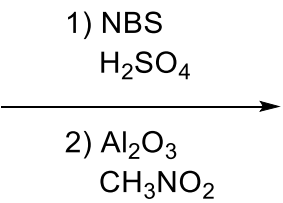

$\mathrm{CH}_{3} \mathrm{NO}_{2}$<smiles>O=[N+]([O-])CC(O)c1c([N+](=O)[O-])cc2c(c1Br)OCO2</smiles>

s1

$29 \%$ (2 steps)<smiles>O=[N+]([O-])CC(O)c1cc2c(c(Br)c1[N+](=O)[O-])OCO2</smiles>

S2

$7.8 \%$ (2 steps)

To a solution of 6-nitropiperonal $(4,30 \mathrm{mg}, 0.15 \mathrm{mmol})$ in concentrated $\mathrm{H}_{2} \mathrm{SO}_{4}(240 \mu \mathrm{L}), \mathrm{N}$ bromosuccinimide (54.8 $\mathrm{mg}, 0.30 \mathrm{mmol}$ ) was slowly added at $\mathrm{rt}$ under Ar atmosphere. The reaction mixture was stirred for $24 \mathrm{~h}$ at $\mathrm{rt}$. The resulting mixture was poured onto crushed ice. The aqueous layer was extracted three times with EtOAc. The combined organic layer was dried over $\mathrm{MgSO}_{4}$, and concentrated under reduced pressure. The residue was filtrated with silica pad with benzene and concentrated under reduced pressure. The residue was dissolved to nitromethane $(420 \mu \mathrm{L})$ and $\mathrm{Al}_{2} \mathrm{O}_{3}$ $(1.7 \mathrm{~g}, 16.7 \mathrm{mmol})$ was added at $\mathrm{rt}$ under Ar atmosphere. The reaction mixture was stirred for $9 \mathrm{~h}$ at $\mathrm{rt}$. The resulting mixture was filtrated with Celite pad with $\mathrm{CHCl}_{3}$. The resulting solution was concentrated under reduced pressure. The crude materials were purified by flash chromatography $\left(\mathrm{SiO}_{2}, 2 \% \mathrm{CH}_{3} \mathrm{CN} / \mathrm{CHCl}_{3}\right)$ to provide $\mathbf{S 1}(15.1 \mathrm{mg}, 29 \%, 2$ steps $)$ as a yellow amorphous powder and S2 (4.0 mg, 7.8\%, 2 steps) as a yellow amorphous powder.

\section{1-(4-Bromo-6-nitrobenzo[d][1,3]dioxol-5-yl)-2-nitroethan-1-ol (S1).}

A yellow amorphous powder; IR (neat) $v_{\max } 3502,1537,1464,1362,1339,1244,1036,932,843 \mathrm{~cm}^{-}$ '; ${ }^{1} \mathrm{H}$ NMR $\left(500 \mathrm{MHz},\left(\mathrm{CD}_{3}\right)_{2} \mathrm{CO}\right) \delta 7.29(\mathrm{~s}, 1 \mathrm{H}), 6.33(\mathrm{~s}, 2 \mathrm{H}), 5.99$ (dd, $\left.J=10.3,2.8 \mathrm{~Hz}, 1 \mathrm{H}\right), 5.77$ (s, 1H), $5.10(\mathrm{dd}, J=13.5,10.3 \mathrm{~Hz}, 1 \mathrm{H}), 4.87(\mathrm{dd}, J=13.5,2.8 \mathrm{~Hz}, 1 \mathrm{H}) ;{ }^{13} \mathrm{C}$ NMR $(125 \mathrm{MHz}$, $\left.\left(\mathrm{CD}_{3}\right)_{2} \mathrm{CO}\right) \delta 149.8,148.2,146.9,126.8,105.3,104.7,101.7,79.4,70.3$; HRESIMS $m / z$ 356.9342 [M $+\mathrm{Na}]^{+}$(calcd for $\mathrm{C}_{9} \mathrm{H}_{7}{ }^{79} \mathrm{BrN}_{2} \mathrm{NaO}_{7}, 356.9329$ ).

\section{1-(7-Bromo-6-nitrobenzo[d][1,3]dioxol-5-yl)-2-nitroethan-1-ol (S2).}

A yellow amorphous powder; IR (neat) $v_{\max } 3503,1530,1470,1362,1339,1256,1040,934,866,812$ $\mathrm{cm}^{-1} ;{ }^{1} \mathrm{H}$ NMR $\left(500 \mathrm{MHz}, \mathrm{CDCl}_{3}\right) \delta 7.10(\mathrm{~s}, 1 \mathrm{H}), 6.19(\mathrm{~s}, 2 \mathrm{H}), 5.42(\mathrm{~d}, J=9.0 \mathrm{~Hz}, 1 \mathrm{H}), 4.66(\mathrm{dd}, J=$ 14.0, $2.3 \mathrm{~Hz}, 1 \mathrm{H}), 4.53(\mathrm{dd}, J=14.0,9.0 \mathrm{~Hz}, 1 \mathrm{H}), 3.11(\mathrm{~d}, J=2.3 \mathrm{~Hz}, 1 \mathrm{H}) ;{ }^{13} \mathrm{C}$ NMR $(125 \mathrm{MHz}$, $\left.\mathrm{CDCl}_{3}\right) \delta 149.6,147.9,143.7,127.5,105.9,103.4,94.4,80.1,66.8$; HRESIMS $m / z$ 356.9345 [M + $\mathrm{Na}]^{+}$(calcd for $\left.\mathrm{C}_{9} \mathrm{H}_{7}{ }^{79} \mathrm{BrN}_{2} \mathrm{NaO}_{7}, 356.9329\right)$.

\section{Synthesis of 4-monobromo indole 9.}


<smiles>O=[N+]([O-])CC(O)c1c([N+](=O)[O-])cc2c(c1Br)OCO2</smiles>

s1 \begin{tabular}{l} 
1) pyridine \\
$\mathrm{Ac}_{2} \mathrm{O}$ \\
\hline
\end{tabular}

2) $\mathrm{Fe}, \mathrm{AcOH}$, silica gel benzene/cyclohexane $56 \%$ (2 steps)<smiles>Brc1c2c(cc3[nH]ccc13)OCO2</smiles>

9

To a solution of $\mathbf{S 1}(33.8 \mathrm{mg}, 0.10 \mathrm{mmol})$ in acetic anhydride $(200 \mu \mathrm{L})$ and pyridine $(45 \mu \mathrm{L}, 0.50$ mmol) was added at $\mathrm{rt}$ under Ar atmosphere. The reaction mixture was stirred for $12 \mathrm{~h}$ at $\mathrm{rt}$. The resulting mixture was quenched with saturated aqueous $\mathrm{NaHCO}_{3}$ solution. The aqueous layer was extracted three times with EtOAc. The combined organic layer was dried over $\mathrm{MgSO}_{4}$, and concentrated under reduced pressure. The residue was dissolved to benzene $(620 \mu \mathrm{L})$ and cyclohexane $(210 \mu \mathrm{L})$, and acetic acid $(550 \mu \mathrm{L})$, silica gel $(91.0 \mathrm{mg})$ and iron $(71.2 \mathrm{mg}, 1.3 \mathrm{mmol})$ were added to the mixture at $\mathrm{rt}$ under Ar atmosphere. The reaction mixture was warmed up to $100{ }^{\circ} \mathrm{C}$, and stirred for $30 \mathrm{~min}$ at $100{ }^{\circ} \mathrm{C}$. After cooling to $\mathrm{rt}$, the resulting mixture was filtrated with Celite pad with $\mathrm{CHCl}_{3}$. The organic layer was washed with saturated brine, saturated $\mathrm{NaHSO}_{3}$ solution and saturated $\mathrm{NaHCO}_{3}$ solution. The combined organic layer was dried over $\mathrm{MgSO}_{4}$, and concentrated under reduced pressure. The crude materials were purified by flash chromatography $\left(\mathrm{SiO}_{2}, 50 \% \mathrm{CHCl}_{3} / n\right.$-hexane $)$ to provide 9 (13.4 mg, 56\%, 2 steps) as pale yellow crystals.

8-Bromo-5H-[1,3]dioxolo[4,5-f]indole (9).

Pale yellow crystals; mp $110-114^{\circ} \mathrm{C}$; IR (neat) $v_{\max } 3401,1458,1296,1034,939,881,849,745,708$ $\mathrm{cm}^{-1} ;{ }^{1} \mathrm{H} \mathrm{NMR}\left(500 \mathrm{MHz}, \mathrm{CDCl}_{3}\right) \delta 8.15$ (br. s, 1H), 7.12 (t, $\left.J=2.7 \mathrm{~Hz}, 1 \mathrm{H}\right), 6.80(\mathrm{~s}, 1 \mathrm{H}), 6.50(\mathrm{t}, J$ $=2.7 \mathrm{~Hz}, 1 \mathrm{H}), 6.01(\mathrm{~s}, 2 \mathrm{H}) ;{ }^{13} \mathrm{C} \mathrm{NMR}\left(125 \mathrm{MHz}, \mathrm{CDCl}_{3}\right) \delta 145.2,141.3,130.4,123.3,122.9,102.8$, 101.2, 92.2, 91.4; HRESIMS $m / z$ 239.9671 [M+ H $]^{+}$(calcd for $\mathrm{C}_{9} \mathrm{H}_{7}{ }^{79} \mathrm{BrNO}_{2}, 239.9655$ ).

Synthesis of 7-monobromo indole $\mathbf{1 0 .}$<smiles>O=[N+]([O-])CC(O)c1cc2c(c(Br)c1[N+](=O)[O-])OCO2</smiles>

S2

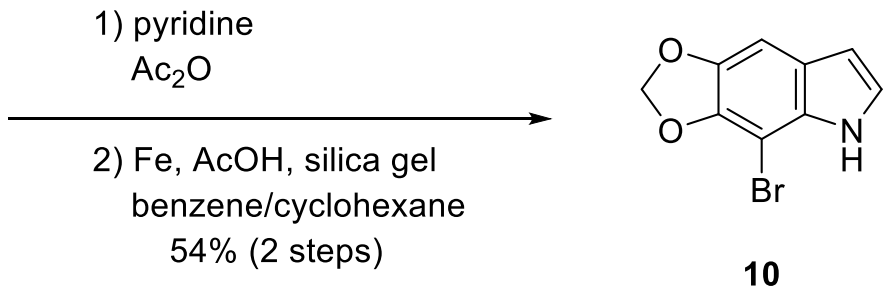

To a solution of $\mathbf{S 2}(34.0 \mathrm{mg}, 0.10 \mathrm{mmol})$ in acetic anhydride $(200 \mu \mathrm{L})$ and pyridine $(45 \mu \mathrm{L}, 0.50$ mmol) was added at $\mathrm{rt}$ under Ar atmosphere. The reaction mixture was stirred for $12 \mathrm{~h}$ at $\mathrm{rt}$. The 
resulting mixture was quenched with saturated aqueous $\mathrm{NaHCO}_{3}$ solution. The aqueous layer was extracted three times with EtOAc. The combined organic layer was dried over $\mathrm{MgSO}_{4}$, and concentrated under reduced pressure. The residue was dissolved to benzene $(620 \mu \mathrm{L})$ and cyclohexane $(210 \mu \mathrm{L})$, and acetic acid $(545 \mu \mathrm{L})$, silica gel $(91.0 \mathrm{mg})$ and iron $(71.2 \mathrm{mg}, 1.3 \mathrm{mmol})$ were added to the mixture at $\mathrm{rt}$ under $\mathrm{Ar}$ atmosphere. The reaction mixture was warmed up to $100^{\circ} \mathrm{C}$, and stirred for 30 min at $100{ }^{\circ} \mathrm{C}$. After cooling to rt, the resulting mixture was filtrated with Celite pad with $\mathrm{CHCl}_{3}$. The organic layer was washed with saturated brine, saturated $\mathrm{NaHSO}_{3}$ solution and saturated $\mathrm{NaHCO}_{3}$ solution. The combined organic layer was dried over $\mathrm{MgSO}_{4}$, and concentrated under reduced pressure. The crude materials were purified by flash chromatography $\left(\mathrm{SiO}_{2}, 50 \% \mathrm{CHCl}_{3} / n\right.$-hexane) to provide 10 (13.4 mg, 56\%, 2 steps) as white crystals.

\section{4-Bromo-5H-[1,3]dioxolo[4,5-f]indole (10).}

White crystals; mp $122-125^{\circ} \mathrm{C}$; IR (neat) $v_{\max } 3397,1460,1306,1032,949,837,754,718 \mathrm{~cm}^{-1} ;{ }^{1} \mathrm{H}$ NMR $\left(500 \mathrm{MHz}, \mathrm{CDCl}_{3}\right) \delta 8.18$ (br. s, 1H), $7.13(\mathrm{t}, J=2.8 \mathrm{~Hz}, 1 \mathrm{H}), 6.97(\mathrm{~s}, 1 \mathrm{H}), 6.50(\mathrm{t}, J=2.8 \mathrm{~Hz}$, 1H), $6.02(\mathrm{~s}, 2 \mathrm{H}) ;{ }^{13} \mathrm{C} \mathrm{NMR}\left(125 \mathrm{MHz}, \mathrm{CDCl}_{3}\right) \delta 143.5,142.8,129.9,123.3,122.1,104.2,101.3,98.7$, 84.3; HRESIMS $m / z 239.9672[\mathrm{M}+\mathrm{H}]^{+}$(calcd for $\mathrm{C}_{9} \mathrm{H}_{7}{ }^{79} \mathrm{BrNO}_{2}, 239.9655$ ).

Dichlorination of 6-nitropiperonal (4).<smiles>O=Cc1cc2c(cc1[N+](=O)[O-])OCO2</smiles>

4

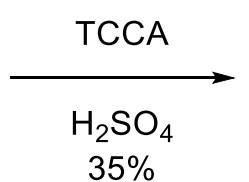

$35 \%$<smiles>O=Cc1c(Cl)c2c(c(Cl)c1[N+](=O)[O-])OCO2</smiles>

S3

To a solution of 6-nitropiperonal $(4,75 \mathrm{mg}, 0.39 \mathrm{mmol})$ in concentrated $\mathrm{H}_{2} \mathrm{SO}_{4}(1.2 \mathrm{~mL})$, trichloroisocyanuric acid (TCCA, $54.8 \mathrm{mg}, 0.30 \mathrm{mmol}$ ) was added at $\mathrm{rt}$ under Ar atmosphere. The reaction mixture was warmed up to $60{ }^{\circ} \mathrm{C}$, and stirred for $12 \mathrm{~h}$ at $60{ }^{\circ} \mathrm{C}$. The resulting mixture was poured onto crushed ice. The aqueous layer was extracted three times with EtOAc. The combined organic layer was dried over $\mathrm{MgSO}_{4}$, and concentrated under reduced pressure. The crude materials were purified by flash chromatography $\left(\mathrm{SiO}_{2}, 50 \% \mathrm{CHCl}_{3} / n\right.$-hexane) to provide $\mathbf{S 3}$ (35 $\left.\mathrm{mg}, 35 \%\right)$ as pale yellow crystals.

\section{4,7-Dichloro-6-nitrobenzo $[d][1,3]$ dioxole-5-carbaldehyde $(\mathbf{S 3})$.}


Pale yellow crystals; $\mathrm{mp} 173-175^{\circ} \mathrm{C}$; IR (neat) $v_{\max } 1697,1543,1456,1339,1260,1032,930,893$, $741718 \mathrm{~cm}^{-1}$; ${ }^{1} \mathrm{H}$ NMR (500 MHz, DMSO-d $) \delta 10.09$ (s, 1H), $6.50(\mathrm{~s}, 2 \mathrm{H}) ;{ }^{13} \mathrm{C}$ NMR (125 MHz, DMSO- $\left.d_{6}\right) \delta 185.0,149.9,147.2,143.0,118.7,114.7,106.3,105.5$; HRESIMS $m / z 285.9298[\mathrm{M}+$ $\mathrm{Na}]^{+}$(calcd for $\mathrm{C}_{8} \mathrm{H}_{3}{ }^{35} \mathrm{Cl}_{2} \mathrm{NNaO}_{5}, 285.9280$ ).

Synthesis of 4,7-dichloro indole 11.<smiles>O=Cc1c(Cl)c2c(c(Cl)c1[N+](=O)[O-])OCO2</smiles>

S3
1) $\mathrm{Al}_{2} \mathrm{O}_{3}, \mathrm{CH}_{3} \mathrm{NO}_{2}$

2) pyridine $\mathrm{Ac}_{2} \mathrm{O}$

3) $\mathrm{Fe}, \mathrm{AcOH}$, silica gel benzene/cyclohexane $51 \%$ (3 steps)<smiles>Clc1c2c(c(Cl)c3[nH]ccc13)OCO2</smiles>

11

To a solution of $\mathbf{S 3}(70 \mathrm{mg}, 0.27 \mathrm{mmol})$ in nitromethane $(1.15 \mathrm{~mL}), \mathrm{Al}_{2} \mathrm{O}_{3}(81.1 \mathrm{mg}, 0.80 \mathrm{mmol})$ was added at rt under Ar atmosphere. The reaction mixture was stirred for $12 \mathrm{~h}$ at $\mathrm{rt}$. The resulting mixture was filtrated with Celite pad and a silica gel pad with $\mathrm{CHCl}_{3}$. The resulting solution was concentrated under reduced pressure. The residue was dissolved to acetic anhydride $(530 \mu \mathrm{L})$ and pyridine $(120 \mu \mathrm{L}$, $1.2 \mathrm{mmol}$ ) was added at $\mathrm{rt}$ under Ar atmosphere. The reaction mixture was stirred for $12 \mathrm{~h}$ at $\mathrm{rt}$. The resulting mixture was quenched with saturated aqueous $\mathrm{NaHCO}_{3}$ solution. The aqueous layer was extracted three times with $\mathrm{CHCl}_{3}$. The combined organic layer was dried over $\mathrm{MgSO}_{4}$, and concentrated under reduced pressure. The residue was dissolved to benzene $(1.8 \mathrm{~mL})$ and cyclohexane $(600 \mu \mathrm{L})$, and acetic acid $(1.6 \mathrm{~mL})$, silica gel $(265 \mathrm{mg})$ and iron $(207 \mathrm{mg}, 3.7 \mathrm{mmol})$ were added to the mixture at rt under $\mathrm{Ar}$ atmosphere. The reaction mixture was warmed up to $100{ }^{\circ} \mathrm{C}$, and stirred for 30 min at $100{ }^{\circ} \mathrm{C}$. After cooling to rt, the resulting mixture was filtrated with Celite pad with $\mathrm{CHCl}_{3}$. The organic layer was washed with saturated brine, saturated $\mathrm{NaHSO}_{3}$ solution, and saturated $\mathrm{NaHCO}_{3}$ solution. The combined organic layer was dried over $\mathrm{MgSO}_{4}$, and concentrated under reduced pressure. The crude materials were purified by flash chromatography $\left(\mathrm{SiO}_{2}, 33 \% \mathrm{CHCl}_{3} / n\right.$-hexane) to provide 11 (31.2 $\mathrm{mg}, 51 \%, 3$ steps) as white crystals.

\section{4,8-Dichloro-5H-[1,3]dioxolo[4,5-f]indole (11).}

White crystals; mp 243-247 ${ }^{\circ} \mathrm{C}$; IR (neat) $v_{\max } 3410,1456,1314,1211,1086,1059,1032,947,866$, 752, $714 \mathrm{~cm}^{-1} ;{ }^{1} \mathrm{H}$ NMR (500 MHz, DMSO- $\left.d_{6}\right) \delta 11.64$ (br. s, $\left.1 \mathrm{H}\right), 7.32(\mathrm{~d}, J=3.3 \mathrm{~Hz}, 1 \mathrm{H}), 6.43$ (d, $J=3.3 \mathrm{~Hz}, 1 \mathrm{H}), 6.15(\mathrm{~s}, 2 \mathrm{H}) ;{ }^{13} \mathrm{C}$ NMR $\left(125 \mathrm{MHz}, \mathrm{DMSO}-d_{6}\right) \delta 140.9,139.2,128.0,125.7,121.1$, 102.3, 102.0, 100.6, 96.0; HRESIMS $m / z 229.9786[\mathrm{M}+\mathrm{H}]^{+}$(calcd for $\mathrm{C}_{9} \mathrm{H}_{6}{ }^{35} \mathrm{Cl}_{2} \mathrm{NO}_{2}, 229.9770$ ). 
Synthesis of $N$-acetyl derivative $\mathbf{1 2}$.<smiles>Brc1c2c(c(Br)c3[nH]ccc13)OCO2</smiles>

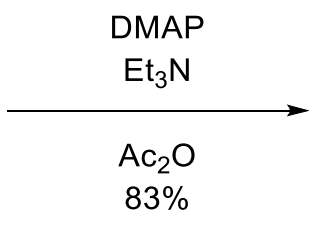

$83 \%$

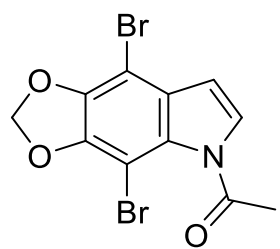

12

To a solution of amakusamine $(1,30 \mathrm{mg}, 0.09 \mathrm{mmol})$ in acetic anhydride $\left(\mathrm{Ac}_{2} \mathrm{O}, 280 \mu \mathrm{L}\right), 4-$ dimethylaminopyridine (DMAP, $1.2 \mathrm{mg}, 0.009 \mathrm{mmol})$ and $\mathrm{Et}_{3} \mathrm{~N}(25 \mu \mathrm{L}, 0.18 \mathrm{mmol})$ were added at $\mathrm{rt}$ under Ar atmosphere. The reaction mixture was stirred for $27 \mathrm{~h}$ at $\mathrm{rt}$. The resulting mixture was quenched with saturated aqueous $\mathrm{NaHCO}_{3}$ solution. The aqueous layer was extracted three times with EtOAc. The combined organic layer was dried over $\mathrm{MgSO}_{4}$, and concentrated under reduced pressure. The crude materials were purified by flash chromatography $\left(\mathrm{SiO}_{2}, 40 \% \mathrm{CHCl}_{3} / n\right.$-hexane) to provide $12(28.2 \mathrm{mg}, 83 \%)$ as white crystals.

\section{1-(4,8-Dibromo-5H-[1,3]dioxolo[4,5-f] indol-5-yl)ethan-1-one (12).}

White crystals; mp $172-175{ }^{\circ} \mathrm{C}$; IR (neat) $v_{\max } 1717,1433,1271,1196,1057,1024,943,837 \mathrm{~cm}^{-1}$; ${ }^{1} \mathrm{H}$ NMR $\left(500 \mathrm{MHz}, \mathrm{CDCl}_{3}\right) \delta 7.36(\mathrm{~d}, J=3.5 \mathrm{~Hz}, 1 \mathrm{H}), 6.60$ (d, J=3.5 Hz, 1H), 6.14 (s, 2H), 2.65 (s, $3 \mathrm{H}) ;{ }^{13} \mathrm{C} \mathrm{NMR}\left(125 \mathrm{MHz}, \mathrm{CDCl}_{3}\right) \delta 166.9,146.0,143.2,129.4,128.7,126.4,108.0,101.9,92.1,89.2$, 24.5; HRESIMS $m / z 381.8710[\mathrm{M}+\mathrm{Na}]^{+}$(calcd for $\mathrm{C}_{11} \mathrm{H}_{7}{ }^{79} \mathrm{Br}_{2} \mathrm{NNaO}_{3}, 381.8685$ ).

Synthesis of $N$-acyl derivative 13-15.<smiles>Brc1c2c(c(Br)c3[nH]ccc13)OCO2</smiles>

1

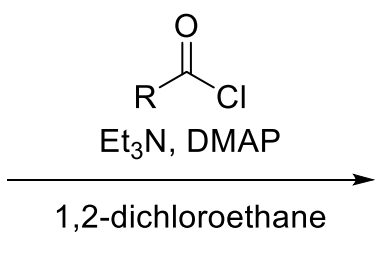

$$
\begin{aligned}
\mathrm{R} & =\mathrm{C}_{3} \mathrm{H}_{7} 13(35 \%) \\
& =\mathrm{C}_{7} \mathrm{H}_{13} 14(56 \%) \\
& =\mathrm{C}_{13} \mathrm{H}_{27} 15(43 \%)
\end{aligned}
$$

To a solution of amakusamine (1) in 1,2-dichloroethane (0.2 M), 4-dimethylaminopyridine (DMAP, 0.1 equiv.), $\mathrm{Et}_{3} \mathrm{~N}$ (2.0 equiv.), and $n$-butyryl chloride [or $n$-octanoyl chloride or myristoyl chloride] (1.5 equiv.) were added at $\mathrm{rt}$ under Ar atmosphere. The reaction mixture was warmed up to $70{ }^{\circ} \mathrm{C}$, and stirred for $15 \mathrm{~h}$ at $70{ }^{\circ} \mathrm{C}$. After cooling to rt, the resulting mixture was quenched with saturated aqueous $\mathrm{NaHCO}_{3}$ solution. The aqueous layer was extracted three times with EtOAc. The combined organic 
layer was dried over $\mathrm{MgSO}_{4}$, and concentrated under reduced pressure. The crude materials were purified by flash chromatography $\left(\mathrm{SiO}_{2}, 35 \% \mathrm{CHCl}_{3} / n\right.$-hexane) to provide $\mathbf{1 3}$ ( $0.09 \mathrm{mmol}$ scale, $35 \%$ ) as white crystals, $14(0.03 \mathrm{mmol}$ scale, $56 \%)$ as white crystals, and $15(0.09 \mathrm{mmol}$ scale, $43 \%)$ as white crystals.

\section{1-(4,8-Dibromo-5H-[1,3]dioxolo[4,5-f]indol-5-yl)butan-1-one (13).}

White crystals; mp 90-92 ${ }^{\circ} \mathrm{C}$; IR (neat) $v_{\max } 2920,1718,1431,1193,1052,946,834,707 \mathrm{~cm}^{-1} ;{ }^{1} \mathrm{H}$ NMR $\left(600 \mathrm{MHz}, \mathrm{CDCl}_{3}\right) \delta 7.38(\mathrm{~d}, J=5.6 \mathrm{~Hz}, 1 \mathrm{H}), 6.62(\mathrm{~d}, J=5.6 \mathrm{~Hz}, 1 \mathrm{H}), 6.14(\mathrm{~s}, 2 \mathrm{H}), 2.90(\mathrm{t}, J$ $=7.2 \mathrm{~Hz}, 2 \mathrm{H}), 1.90$ (sext., $J=7.2 \mathrm{~Hz}, 2 \mathrm{H}), 1.01(\mathrm{t}, J=7.2 \mathrm{~Hz}, 3 \mathrm{H}) ;{ }^{13} \mathrm{C} \mathrm{NMR}\left(150 \mathrm{MHz}, \mathrm{CDCl}_{3}\right) \delta$ 170.3, 145.9, 143.2, 129.5, 128.7, 126.1, 107.8, 101.9, 92.1, 89.2, 38.8, 18.9, 13.8; HRESIMS m/z $387.9146[\mathrm{M}+\mathrm{H}]^{+}$(calcd for $\mathrm{C}_{13} \mathrm{H}_{12}{ }^{79} \mathrm{Br}_{2} \mathrm{NO}_{3}, 387.9178$ ).

1-(4,8-Dibromo-5H-[1,3]dioxolo[4,5-f]indol-5-yl)octan-1-one (14).

White crystals; $\mathrm{mp} 74-76^{\circ} \mathrm{C}$; IR (neat) $v_{\max } 1722,1423,1339,1306,1244,1188,1053,1034,945$, $837,710 \mathrm{~cm}^{-1} ;{ }^{1} \mathrm{H}$ NMR $\left(500 \mathrm{MHz}, \mathrm{CDCl}_{3}\right) \delta 7.38(\mathrm{~d}, J=3.8 \mathrm{~Hz}, 1 \mathrm{H}), 6.61(\mathrm{~d}, J=3.8 \mathrm{~Hz}, 1 \mathrm{H}), 6.14$ (s, 2H), 2.90 (t, $J=7.3 \mathrm{~Hz}, 2 \mathrm{H}$ ), 1.86 (quint., $J=7.3 \mathrm{~Hz}, 2 \mathrm{H}$ ), 1.45 (quint., $J=7.3 \mathrm{~Hz}, 2 \mathrm{H}$ ), 1.36 (quint., $J=7.3 \mathrm{~Hz}, 2 \mathrm{H}), 1.32-1.29(\mathrm{~m}, 4 \mathrm{H}), 0.89(\mathrm{t}, J=6.8 \mathrm{~Hz}, 3 \mathrm{H}) ;{ }^{13} \mathrm{C} \mathrm{NMR}\left(125 \mathrm{MHz}, \mathrm{CDCl}_{3}\right) \delta$ $170.5,145.8,143.1,129.5,128.6,126.1,107.7,101.9,92.1,89.2,37.0,31.8,29.2,29.1,25.4,22.7$, 14.2; HRESIMS $m / z 465.9650[\mathrm{M}+\mathrm{Na}]^{+}$(calcd for $\mathrm{C}_{17} \mathrm{H}_{19}{ }^{79} \mathrm{Br}_{2} \mathrm{NNaO}_{3}$, 465.9624).

\section{1-(4,8-Dibromo-5H-[1,3]dioxolo[4,5-f]indol-5-yl)tetradecan-1-one (15).}

White crystals; mp $73-75^{\circ} \mathrm{C}$; IR (neat) $v_{\max } 2916,2848,1725,1434,1187,1066,951,839,700 \mathrm{~cm}^{-}$ 1; ${ }^{1} \mathrm{H}$ NMR (600 MHz, $\left.\mathrm{CDCl}_{3}\right) \delta 7.38(\mathrm{~d}, J=3.8 \mathrm{~Hz}, 1 \mathrm{H}), 6.61(\mathrm{~d}, J=3.8 \mathrm{~Hz}, 1 \mathrm{H}), 6.14(\mathrm{~s}, 2 \mathrm{H}), 2.90$ (t, $J=7.3 \mathrm{~Hz}, 2 \mathrm{H}), 1.86$ (quint., $J=7.3 \mathrm{~Hz}, 2 \mathrm{H}), 1.47-1.42$ (m, 2H), 1.37-1.34 (m, 2H), 1.31-1.22 (m, 16H), $0.88(\mathrm{t}, J=7.3 \mathrm{~Hz}, 3 \mathrm{H}) ;{ }^{13} \mathrm{C}$ NMR $\left(150 \mathrm{MHz}, \mathrm{CDCl}_{3}\right) \delta 170.5,145.9,143.1,129.5,128.6$, 126.1, 107.7, 101.9, 92.1, 89.2, 37.0, 32.1, 29.8 (3C), 29.7, 29.6, 29.5, 29.4, 29.2, 25.4, 22.8, 14.3; HRESIMS $m / z 550.0567[\mathrm{M}+\mathrm{Na}]^{+}$(calcd for $\mathrm{C}_{23} \mathrm{H}_{31}{ }^{79} \mathrm{Br}_{2} \mathrm{NNaO}_{3}, 550.0563$ ).

Synthesis of $N$-benzoyl derivative 16. 
<smiles>Brc1c2c(c(Br)c3[nH]ccc13)OCO2</smiles>

1

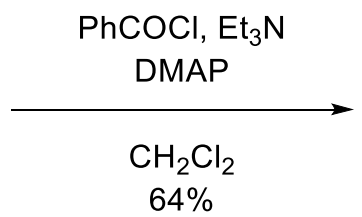

$64 \%$<smiles>O=C(c1ccccc1)n1ccc2c(Br)c3c(c(Br)c21)OCO3</smiles>

16

To a solution of amakusamine $(1,15 \mathrm{mg}, 0.05 \mathrm{mmol})$ in $\mathrm{CH}_{2} \mathrm{Cl}_{2}(500 \mu \mathrm{L})$, 4-dimethylaminopyridine (DMAP, $0.6 \mathrm{mg}, 0.005 \mathrm{mmol}), \mathrm{Et}_{3} \mathrm{~N}(13 \mu \mathrm{L}, 0.10 \mathrm{mmol})$, and benzoylchloride $(6.6 \mu \mathrm{L})$ were added at $\mathrm{rt}$ under Ar atmosphere. The reaction mixture was stirred for $12 \mathrm{~h}$ at $\mathrm{rt}$. The resulting mixture was quenched with saturated aqueous $\mathrm{NH}_{4} \mathrm{Cl}$ solution. The aqueous layer was extracted three times with EtOAc. The combined organic layer was dried over $\mathrm{MgSO}_{4}$, and concentrated under reduced pressure. The crude materials were purified by flash chromatography $\left(\mathrm{SiO}_{2}, 50 \%\right.$ benzene $/ n$-hexane) to provide 16 (12.6 mg, 64\%) as white crystals.

(4,8-Dibromo-5H-[1,3]dioxolo[4,5-f]indol-5-yl)(phenyl)methanone (16).

White crystals; $m p 160-165^{\circ} \mathrm{C}$; IR (neat) $v_{\max } 1703,1437,1341,1285,1198,1063,1040,945,856$, $837 \mathrm{~cm}^{-1} ;{ }^{1} \mathrm{H}$ NMR $\left(500 \mathrm{MHz}\right.$, DMSO- $\left.d_{6}\right) \delta 7.91(\mathrm{~d}, J=7.5 \mathrm{~Hz}, 2 \mathrm{H}), 7.79(\mathrm{t}, J=7.5 \mathrm{~Hz}, 1 \mathrm{H}), 7.64(\mathrm{t}$, $J=7.5 \mathrm{~Hz}, 2 \mathrm{H}), 7.44(\mathrm{~d}, J=3.3 \mathrm{~Hz}, 1 \mathrm{H}), 6.63(\mathrm{~d}, J=3.3 \mathrm{~Hz}, 1 \mathrm{H}), 6.26(\mathrm{~s}, 2 \mathrm{H}),{ }^{13} \mathrm{C} \mathrm{NMR}(125 \mathrm{MHz}$, DMSO- $\left.d_{6}\right) \delta 166.2,145.2,143.0,134.4,132.2,130.5,130.0,129.3,129.2,127.4,106.3,102.3,91.7$, 87.9; HRESIMS $m / z 443.8861[\mathrm{M}+\mathrm{Na}]^{+}$(calcd for $\left.\mathrm{C}_{16} \mathrm{H}_{9}{ }^{79} \mathrm{Br}_{2} \mathrm{NNaO}_{3}, 443.8841\right)$.

Synthesis of 3-formyl derivative 19.<smiles>Brc1c2c(c(Br)c3[nH]ccc13)OCO2</smiles>

1

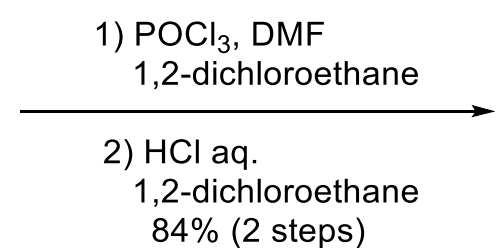

$84 \%$ (2 steps)<smiles>O=Cc1c[nH]c2c(Br)c3c(c(Br)c12)OCO3</smiles>

19

To a solution of DMF ( $52 \mu \mathrm{L}, 0.64 \mathrm{mmol})$, phosphoryl chloride $(59 \mu \mathrm{L}, 0.64 \mathrm{mmol})$ was added at $0{ }^{\circ} \mathrm{C}$ under Ar atmosphere. After the reaction mixture was stirred for $10 \mathrm{~min}$ at $0{ }^{\circ} \mathrm{C}$, dichloromethane $(800$ $\mu \mathrm{L}$ ) was added to the resulting mixture at $0{ }^{\circ} \mathrm{C}$. The reaction mixture was warmed up to rt, and stirred for additional $1 \mathrm{~h}$ at $\mathrm{rt}$. To the resulting mixture, amakusamine $(1,100 \mathrm{mg}, 0.32 \mathrm{mmol})$ was added under $\mathrm{Ar}$ atmosphere. The reaction mixture was stirred for $1 \mathrm{~h}$ at $\mathrm{rt}$. A solution of $\mathrm{NaOH}(1 \mathrm{M}, 1 \mathrm{~mL})$ and 1,2-dichloroethane $(1 \mathrm{~mL})$ was added and the resulting mixture was stirred for additional $1 \mathrm{~h}$ at $80{ }^{\circ} \mathrm{C}$. To the resulting mixture, excess amount of $\mathrm{H}_{2} \mathrm{O}$ was added. The aqueous layer was extracted 
three times with $\mathrm{CHCl}_{3}$. The combined organic layer was dried over $\mathrm{MgSO}_{4}$, and concentrated under reduced pressure. The residue was dissolved to 1,2-dichloroethane $(1 \mathrm{~mL})$ and aqueous $1 \mathrm{M} \mathrm{HCl}$ solution $(1 \mathrm{~mL})$ was added to the mixture at $\mathrm{rt}$ under Ar atmosphere. The reaction mixture was warmed up to $80{ }^{\circ} \mathrm{C}$, and stirred for $2 \mathrm{~h}$ at $80^{\circ} \mathrm{C}$. After cooling to $\mathrm{rt}$, the resulting mixture was basified by slowly adding saturated aqueous $\mathrm{NaHCO}_{3}$ solution. After 1,2-dichloroethane was removed under reduced pressure, the resulting solid was collected by vacuum filtration, washed with water and $n$ hexane. Desired 19 (93 mg, 84\%, 2 steps) was obtained as white crystals.

\section{4,8-Dibromo-5H-[1,3]dioxolo[4,5-f]indole-7-carbaldehyde (19).}

White crystals; mp $280-285^{\circ} \mathrm{C}$; IR (neat) $v_{\max } 3307,1657,1497,1447,1362,1314,1258,1067,1026$, 951, $843 \mathrm{~cm}^{-1} ;{ }^{1} \mathrm{H}$ NMR (500 MHz, DMSO-d $)_{6} \delta 10.52(\mathrm{~s}, 1 \mathrm{H}), 8.07$ (s, 1H), $6.22(\mathrm{~s}, 2 \mathrm{H}), \mathrm{N} \underline{\mathrm{H}}$ was not observed.; ${ }^{13} \mathrm{C}$ NMR $\left(125 \mathrm{MHz}\right.$, DMSO- $\left.d_{6}\right) \delta 184.2,143.1,142.9,133.0,131.4,119.7,119.5$, 102.1, 90.0, 84.8; HRESIMS $m / z 345.8725[\mathrm{M}+\mathrm{H}]^{+}$(calcd for $\mathrm{C}_{10} \mathrm{H}_{6}{ }^{79} \mathrm{Br}_{2} \mathrm{NO}_{3}, 345.8709$ ).

Synthesis of 3-methyl derivative 17.<smiles>O=Cc1c[nH]c2c(Br)c3c(c(Br)c12)OCO3</smiles>

19

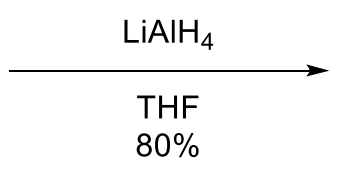

$80 \%$<smiles>Cc1c[nH]c2c(Br)c3c(c(Br)c12)OCO3</smiles>

17

To a solution of $19(10 \mathrm{mg}, 0.03 \mathrm{mmol})$ in dry THF (300 $\mu \mathrm{L})$, lithium aluminum hydride $(2.2 \mathrm{mg}, 0.06$ mmol) was added at $0{ }^{\circ} \mathrm{C}$ under Ar atmosphere. The reaction mixture was warmed up to rt, and stirred for $1 \mathrm{~h}$ at $\mathrm{rt}$. A saturated aqueous Rochelle salt solution was added to the reaction mixture. The resulting mixture was stirred for $12 \mathrm{~h}$ at $\mathrm{rt}$. The aqueous layer was extracted three times with EtOAc. The combined organic layer was dried over $\mathrm{MgSO}_{4}$, and concentrated under reduced pressure. The crude materials were purified by flash chromatography $\left(\mathrm{SiO}_{2}, 25 \% \mathrm{EtOAc} / n\right.$-hexane) to provide 17 ( $7.7 \mathrm{mg}$, $80 \%)$ as white crystals.

\section{4,8-Dibromo-7-methyl-5H-[1,3]dioxolo[4,5-f]indole (17).}

White crystals; mp $185-187^{\circ} \mathrm{C}$; IR (neat) $v_{\max } 3388,2921,2852,1455,1303,1287,1069,1033,946$, 836, $777 \mathrm{~cm}^{-1} ;{ }^{1} \mathrm{H}$ NMR $\left(600 \mathrm{MHz}, \mathrm{CDCl}_{3}\right) \delta 7.96$ (br. s, $\left.1 \mathrm{H}\right), 6.91$ (s, 1H), 6.08 (s, 2H), $2.48(\mathrm{~s}, 3 \mathrm{H})$; ${ }^{13} \mathrm{C}$ NMR $\left(150 \mathrm{MHz}, \mathrm{CDCl}_{3}\right) \delta 142.4,141.3,130,9,122.4,120.4,114.8,101.5,92.2,83.8,12.4$; HRESIMS $m / z 331.8922[\mathrm{M}+\mathrm{H}]^{+}$(calcd for $\mathrm{C}_{10} \mathrm{H}_{8}{ }^{79} \mathrm{Br}_{2} \mathrm{NO}_{2}, 331.8916$ ). 
Synthesis of 3-hydroxymethyl derivative $\mathbf{1 8 .}$<smiles>O=Cc1c[nH]c2c(Br)c3c(c(Br)c12)OCO3</smiles>

19

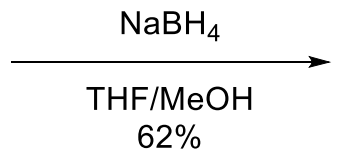

$62 \%$<smiles>OCc1c[nH]c2c(Br)c3c(c(Br)c12)OCO3</smiles>

18

To a solution of $19(10 \mathrm{mg}, 0.03 \mathrm{mmol})$ in THF $(150 \mu \mathrm{L})$ and $\mathrm{MeOH}(150 \mu \mathrm{L})$, sodium borohydride ( $3.3 \mathrm{mg}, 0.09 \mathrm{mmol}$ ) was added at $\mathrm{rt}$ under Ar atmosphere. The reaction mixture was stirred for $1 \mathrm{~h}$ at rt. The reaction mixture was quenched with saturated aqueous $\mathrm{NH}_{4} \mathrm{Cl}$ solution. The aqueous layer was extracted three times with $\mathrm{CHCl}_{3}$. The combined organic layer was dried over $\mathrm{MgSO}_{4}$, and concentrated under reduced pressure. The crude materials were purified by flash chromatography $\left(\mathrm{SiO}_{2}, 40 \% \mathrm{EtOAc} / n\right.$-hexane) to provide $\mathbf{1 8}(7.7 \mathrm{mg}, 80 \%)$ as white crystals.

\section{(4,8-Dibromo-5H-[1,3]dioxolo[4,5-f]indol-7-yl)methanol (18).}

White crystals; mp $183-186^{\circ} \mathrm{C}$; IR (neat) $v_{\max } 3409,1444,1306,1065,943,899,834,795,701,687$ $\mathrm{cm}^{-1}$; ${ }^{1} \mathrm{H}$ NMR $\left(600 \mathrm{MHz}, \mathrm{CD}_{3} \mathrm{OD}\right) \delta 7.22(\mathrm{~s}, 1 \mathrm{H}), 6.05(\mathrm{~s}, 2 \mathrm{H}), 4.92(\mathrm{~s}, 2 \mathrm{H}), \mathrm{N} \underline{\mathrm{H}}, \mathrm{O} \underline{\mathrm{H}}$ were not observed; ${ }^{13} \mathrm{C}$ NMR $\left(150 \mathrm{MHz}, \mathrm{CD}_{3} \mathrm{OD}\right) \delta 143.7,142.6,132.4,125.9,120.7,118.8,102.7,91.9,84.7$, 57.2; HRESIMS $m / z 347.8854[\mathrm{M}+\mathrm{H}]^{+}\left(\right.$calcd for $\left.\mathrm{C}_{10} \mathrm{H}_{8}{ }^{79} \mathrm{Br}_{2} \mathrm{NO}_{3}, 347.8865\right)$.

Synthesis of tryptamine derivative $\mathbf{2 0}$.<smiles>O=Cc1c[nH]c2c(Br)c3c(c(Br)c12)OCO3</smiles>

19

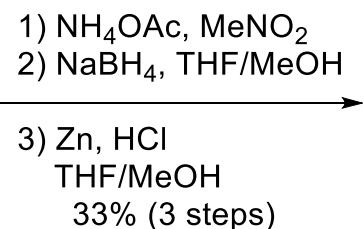
$33 \%$ (3 steps)<smiles>NCCc1c[nH]c2c(Br)c3c(c(Br)c12)OCO3</smiles>

20

To a solution of $19(30 \mathrm{mg}, 0.09 \mathrm{mmol})$ in nitromethane $(500 \mu \mathrm{L}), \mathrm{NH}_{4} \mathrm{OAc}(27 \mathrm{mg}, 0.36 \mathrm{mmol})$ was added at $\mathrm{rt}$ under $\mathrm{Ar}$ atmosphere. The reaction mixture was warmed up to $100{ }^{\circ} \mathrm{C}$, and stirred for 2.5 h at $100{ }^{\circ} \mathrm{C}$. The reaction mixture was quenched with $\mathrm{H}_{2} \mathrm{O}$. The aqueous layer was extracted three times with EtOAc. The combined organic layer was dried over $\mathrm{MgSO}_{4}$, and concentrated under reduced pressure. The residue was dissolved to THF $(400 \mu \mathrm{L})$ and $\mathrm{MeOH}(400 \mu \mathrm{L})$ and sodium borohydride $(9.8 \mathrm{mg}, 0.26 \mathrm{mmol})$ was added to the mixture at $\mathrm{rt}$ under Ar atmosphere. The reaction mixture was stirred for $30 \mathrm{~min}$ at $\mathrm{rt}$. The reaction mixture was quenched with saturated aqueous $\mathrm{NH}_{4} \mathrm{Cl}$ 
solution. The aqueous layer was extracted three times with EtOAc. The combined organic layer was dried over $\mathrm{MgSO}_{4}$, and concentrated under reduced pressure. The residue was filtrated with Celite pad and a silica gel pad with $\mathrm{CHCl}_{3}$. The filtrate was concentrated under reduced pressure.

The crude materials of nitro compound were dissolved to THF $(180 \mu \mathrm{L})$ and $\mathrm{MeOH}(180 \mu \mathrm{L})$, and aqueous $2 \mathrm{M} \mathrm{HCl}$ solution $(2 \mathrm{M}, 360 \mu \mathrm{L})$ and zinc dust (45 mg, $0.69 \mathrm{mmol})$ were added at $\mathrm{rt}$ under $\mathrm{Ar}$ atmosphere. The reaction mixture was warmed up to $70{ }^{\circ} \mathrm{C}$, and stirred for $3 \mathrm{~h}$ at $70{ }^{\circ} \mathrm{C}$. The reaction mixture was quenched with saturated aqueous $\mathrm{NaHCO}_{3}$ solution. The aqueous layer was extracted three times with EtOAc. The combined organic layer was dried over $\mathrm{MgSO}_{4}$, and concentrated under reduced pressure. The crude materials were purified by PTLC $\left(\mathrm{SiO}_{2}-\mathrm{NH}, 10 \% \mathrm{MeOH} / \mathrm{CHCl}_{3}\right)$ to provide 20 (10.3 $\mathrm{mg}, 33 \%, 3$ steps) as white crystals.

\section{2-(4,8-Dibromo-5H-[1,3]dioxolo[4,5-f]indol-7-yl)ethan-1-amine (20)}

White crystals; $\mathrm{mp} 192-193^{\circ} \mathrm{C}$; IR (neat) $v_{\max } 3355,2919,2852,1451,1306,1065,1040,950,937$, $779 \mathrm{~cm}^{-1} ;{ }^{1} \mathrm{H}$ NMR $\left(600 \mathrm{MHz}, \mathrm{CD}_{3} \mathrm{OD}\right) \delta 7.07$ (s, 1H), 6.04 (s, 2H), 3.09 (t, $\left.J=7.2 \mathrm{~Hz}, 2 \mathrm{H}\right), 2.99$ (t, $J=7.2 \mathrm{~Hz}, 2 \mathrm{H}), \mathrm{NH}$ and $\mathrm{NH}_{2}$ were not observed.; ${ }^{13} \mathrm{C} \mathrm{NMR}\left(150 \mathrm{MHz}, \mathrm{CD}_{3} \mathrm{OD}\right) \delta 143.6,142.4,132.5$, 125.2, 120.6, 115.5, 102.7, 91.9, 84.8, 44.1, 29.6; HRESIMS $m / z$ 360.9209 $[\mathrm{M}+\mathrm{H}]^{+}$(calcd for $\left.\mathrm{C}_{11} \mathrm{H}_{11}{ }^{79} \mathrm{Br}_{2} \mathrm{~N}_{2} \mathrm{O}_{2}, 360.9181\right)$.

\section{Reference}

1. Huleatt, P. B.; Lau, J.; Chua, S.; Tan, Y. L.; Doung, H. A.; Chai, C. L. L. Concise, efficient and practical assembly of bromo-5,6-dimethoxyindole building blocks. Tetrahedron Lett. 2011, 52, 13391342. 


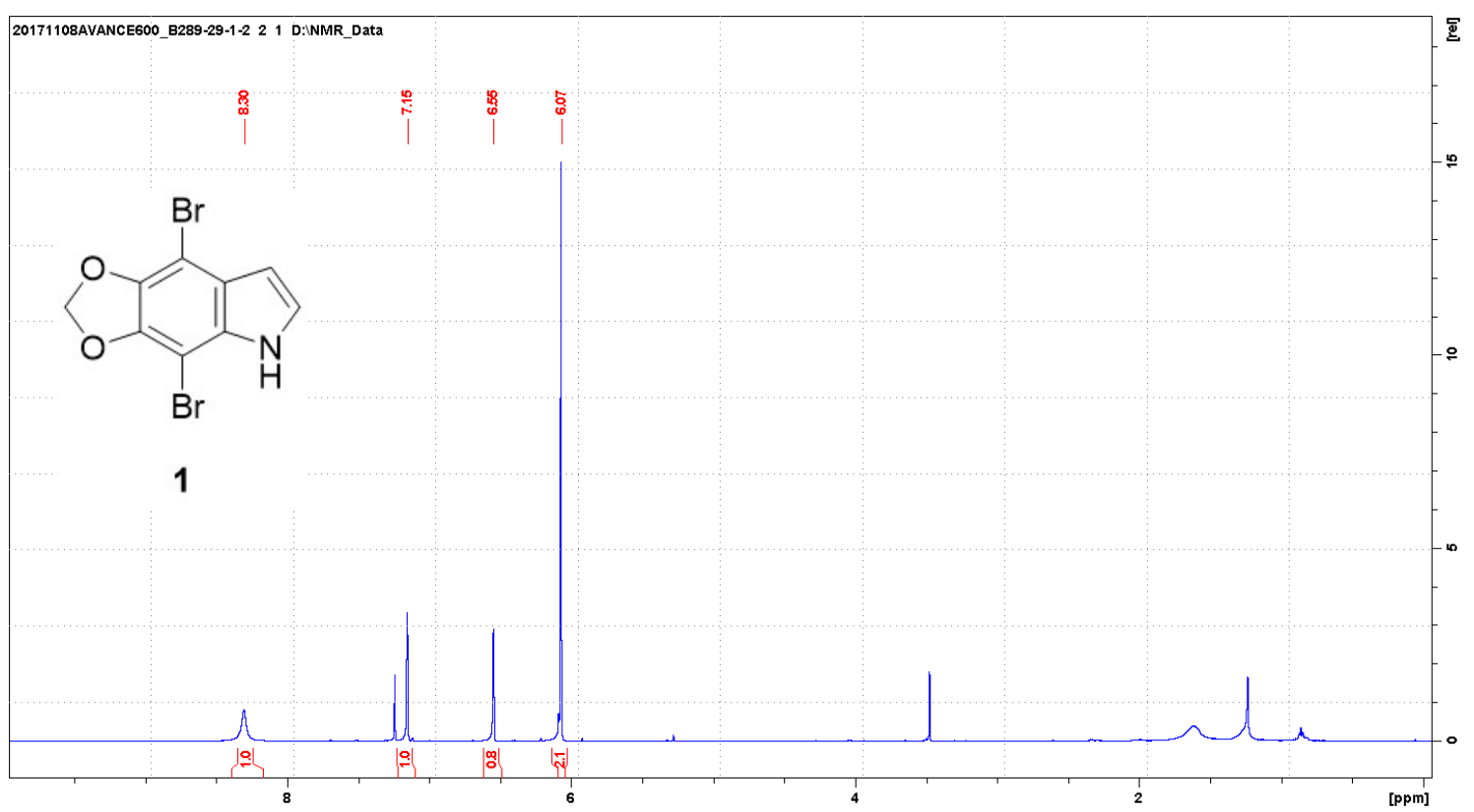

Figure S1. ${ }^{1} \mathrm{H}$ NMR spectrum of natural 1.

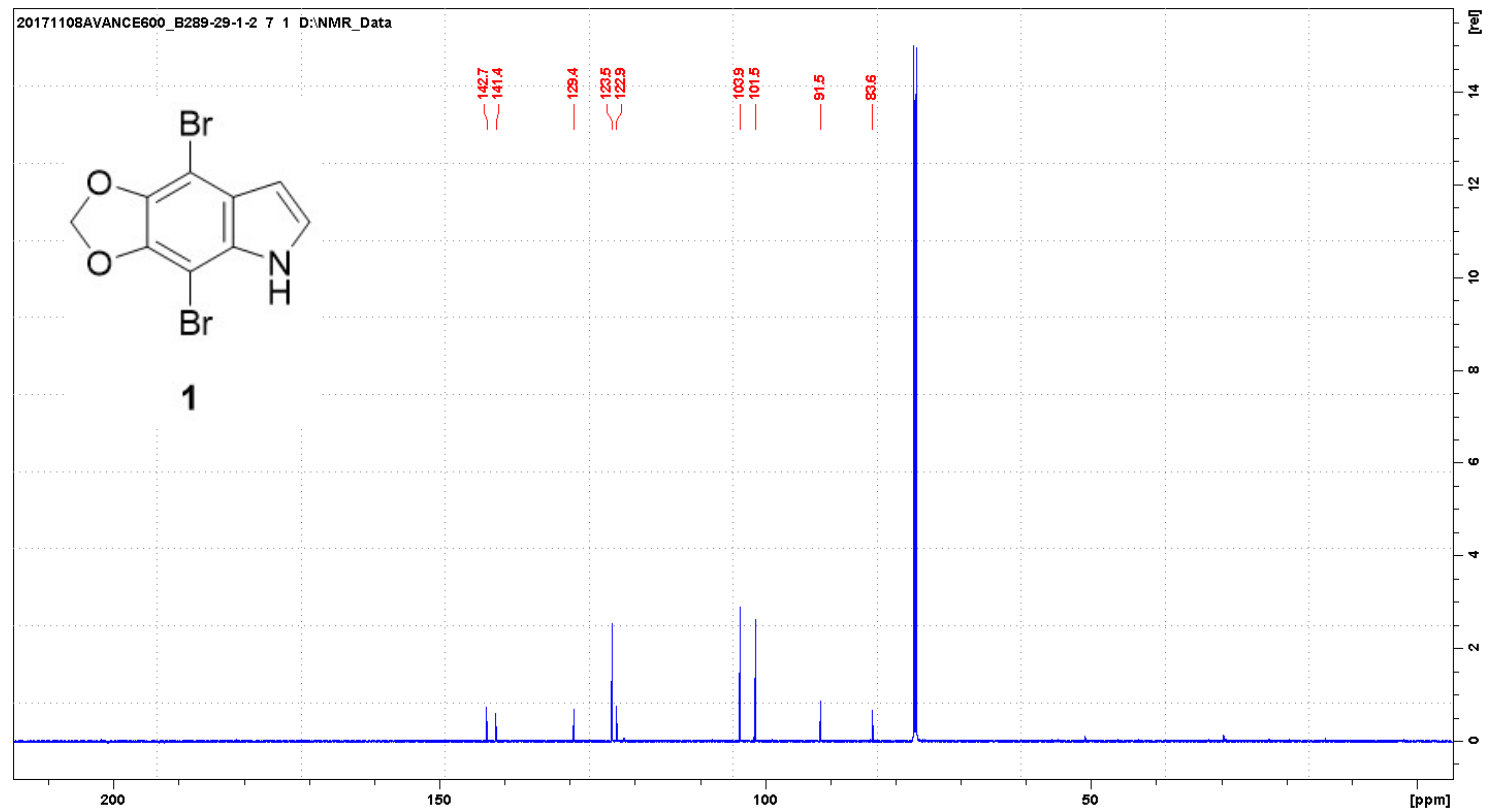

Figure S2. ${ }^{13} \mathrm{C}$ NMR spectrum of natural 1. 


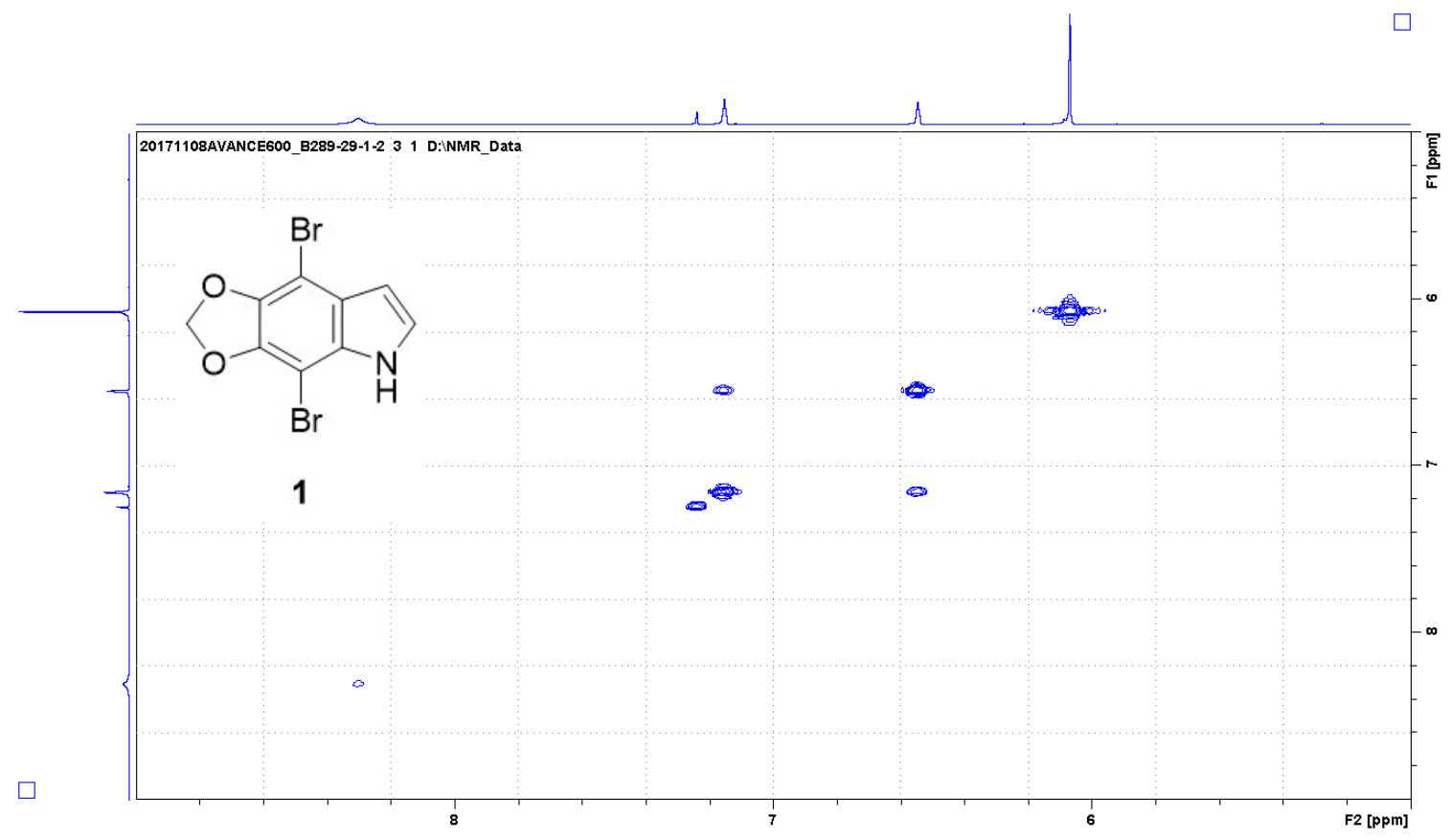

Figure S3. COSY spectrum of natural 1.

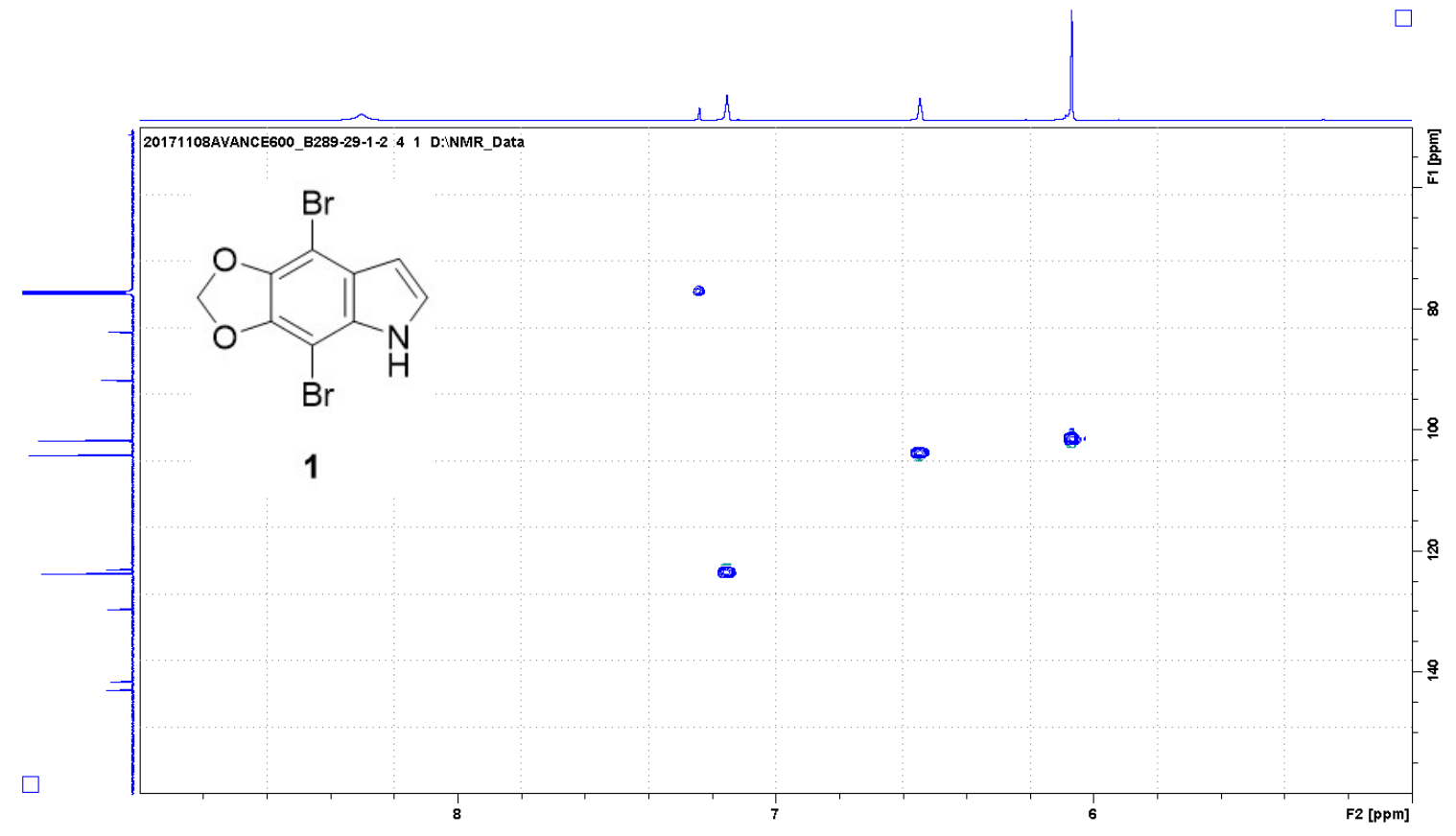

Figure S4. HSQC spectrum of natural 1. 


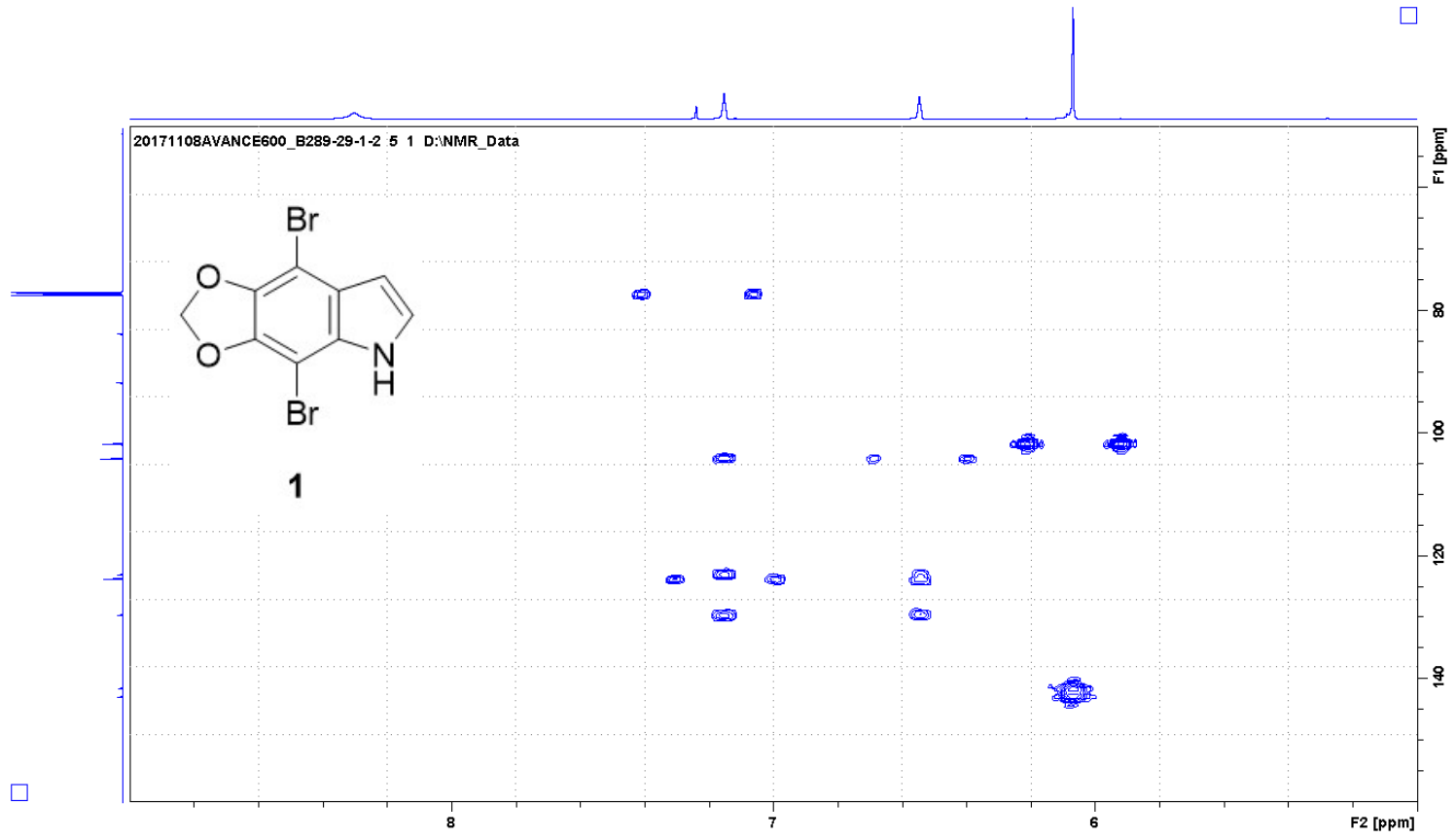

Figure S5. HMBC spectrum of natural 1. 

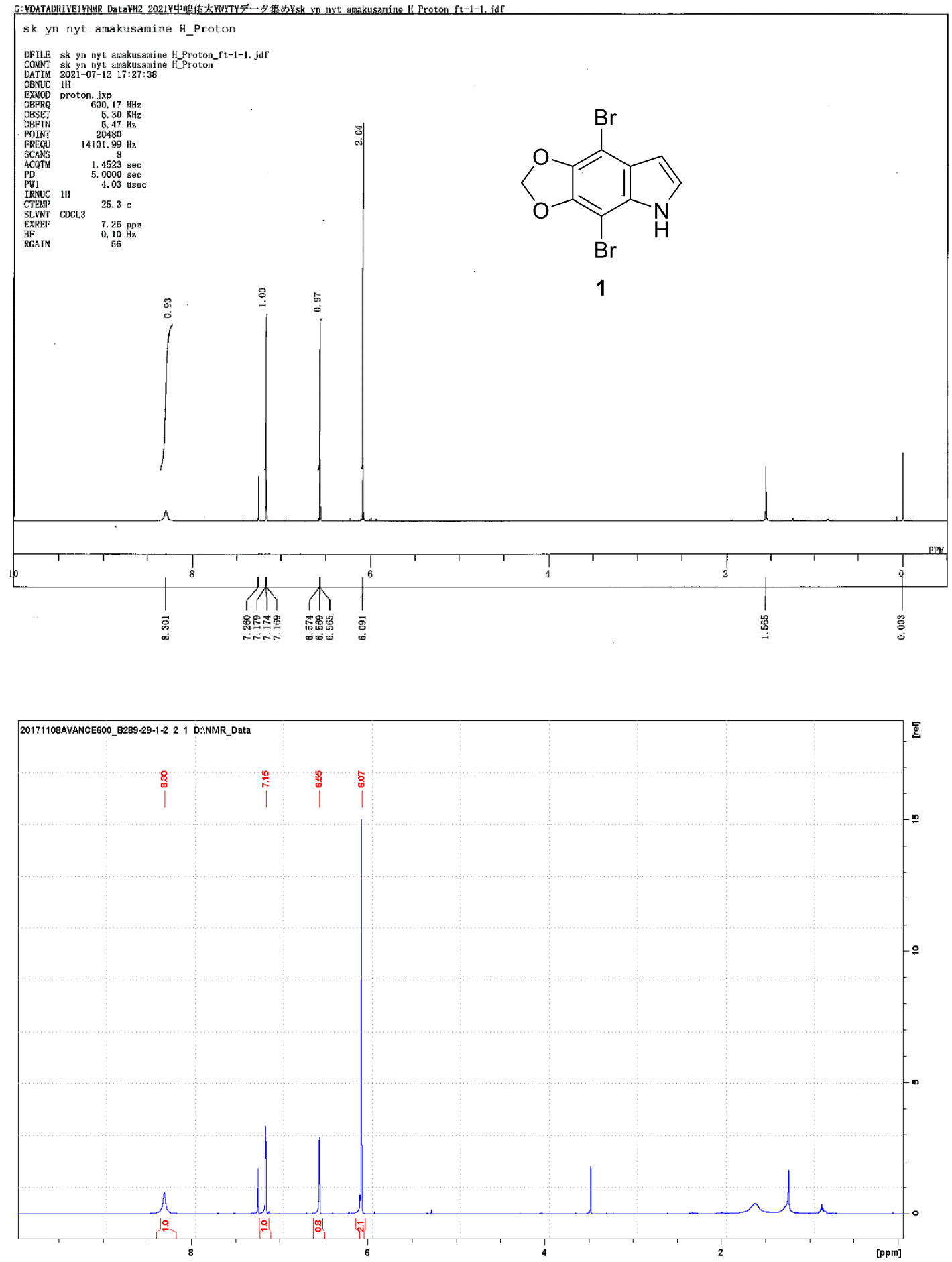

Figure S6. ${ }^{1} \mathrm{H}$ NMR spectra of synthetic (upper) and natural (lower) $\mathbf{1}$. 

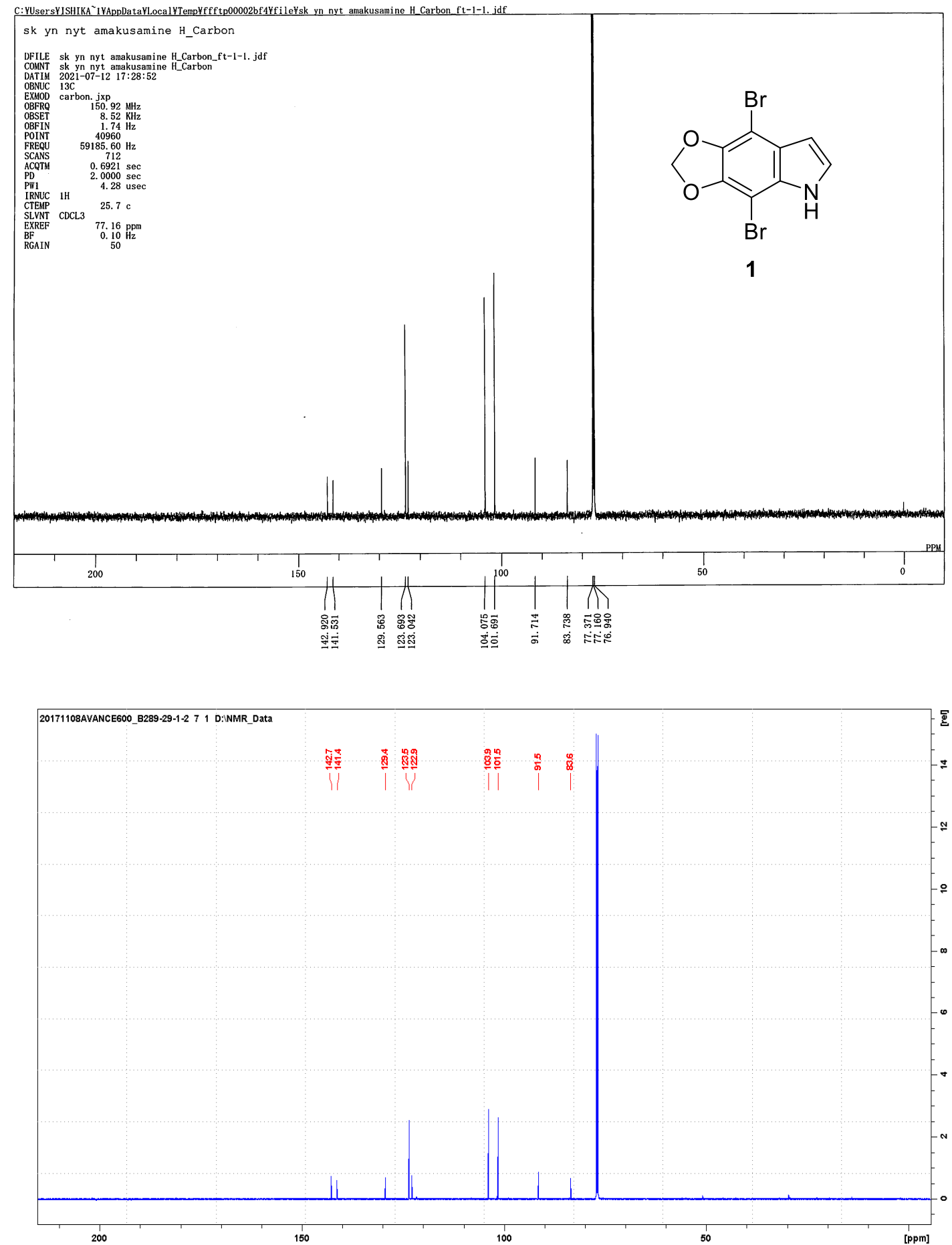

Figure S7. ${ }^{13} \mathrm{C}$ NMR spectra of synthetic (upper) and natural (lower) 1. 

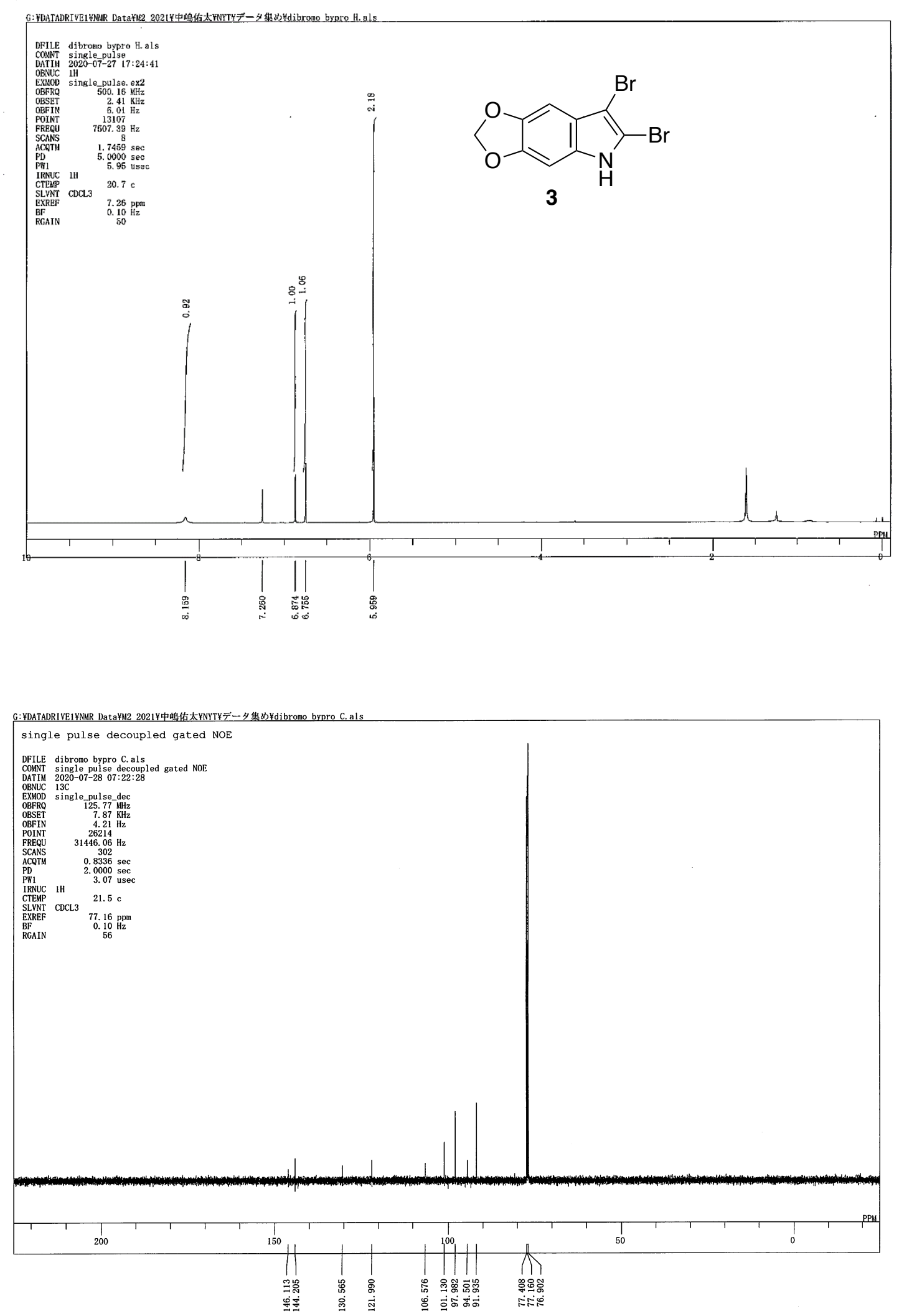

Figure S8. ${ }^{1} \mathrm{H}$ and ${ }^{13} \mathrm{C}$ NMR spectra of 3 . 

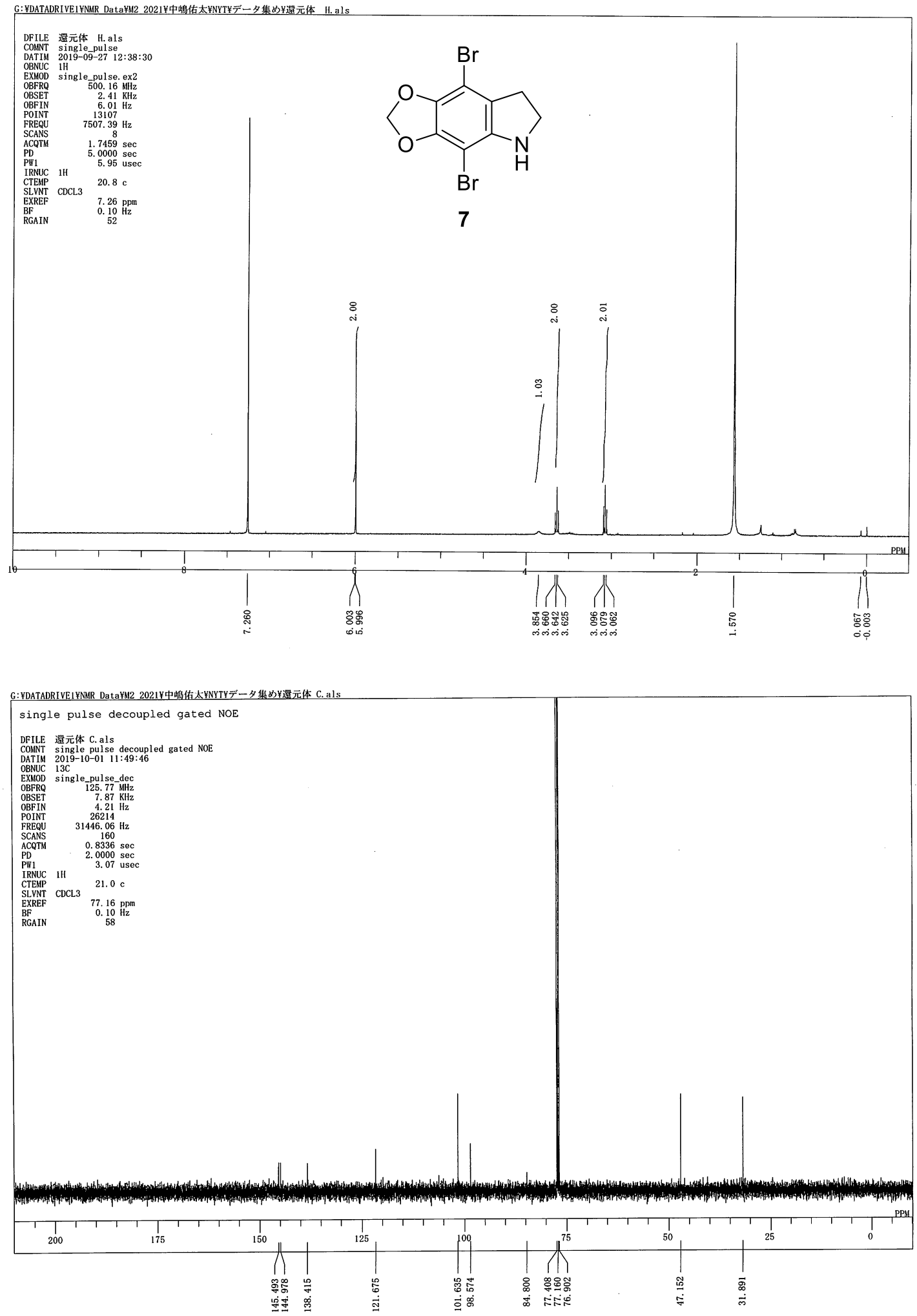

Figure S9. ${ }^{1} \mathrm{H}$ and ${ }^{13} \mathrm{C}$ NMR spectra of 7 . 

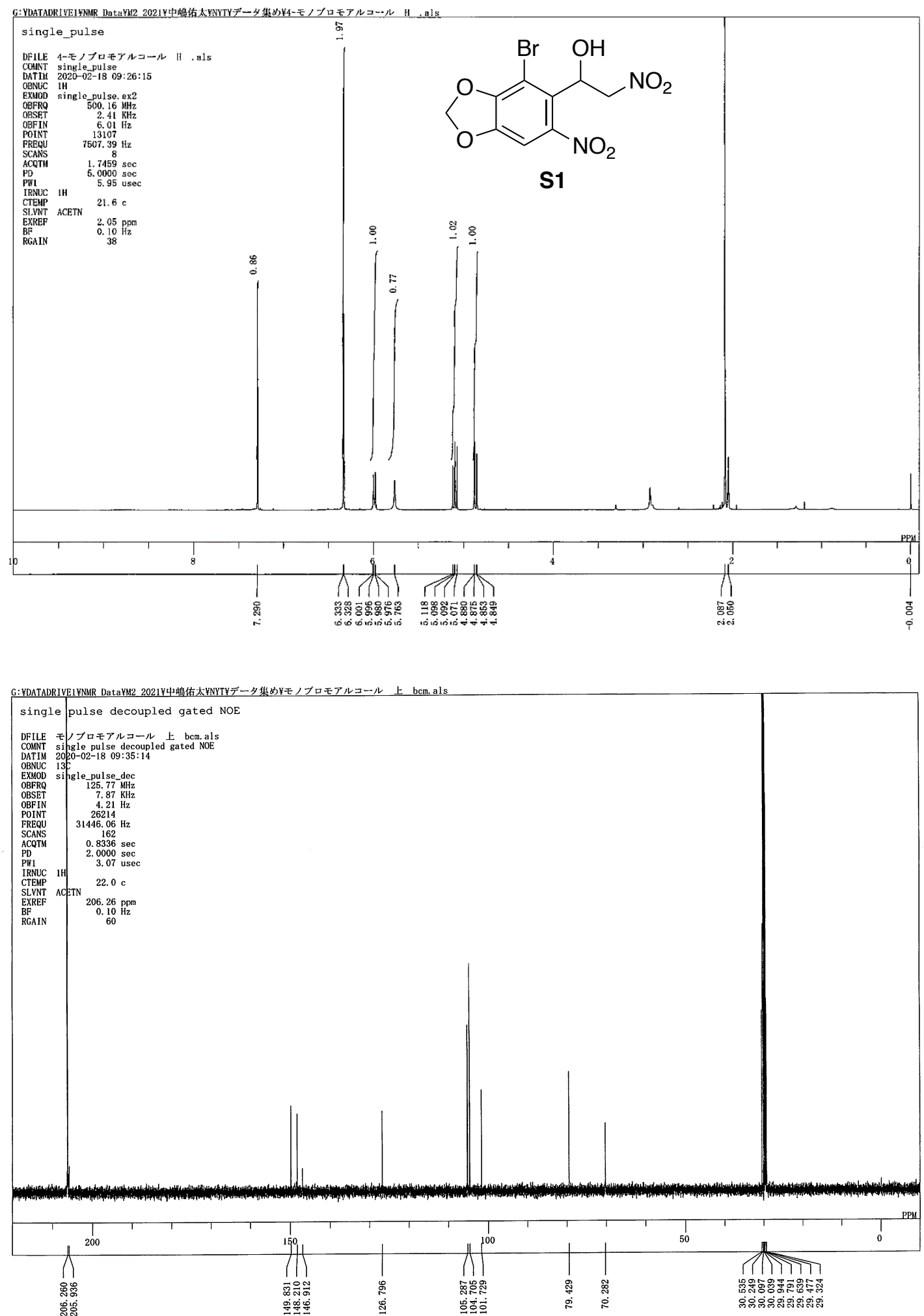

Figure S10. ${ }^{1} \mathrm{H}$ and ${ }^{13} \mathrm{C}$ NMR spectra of S1. 

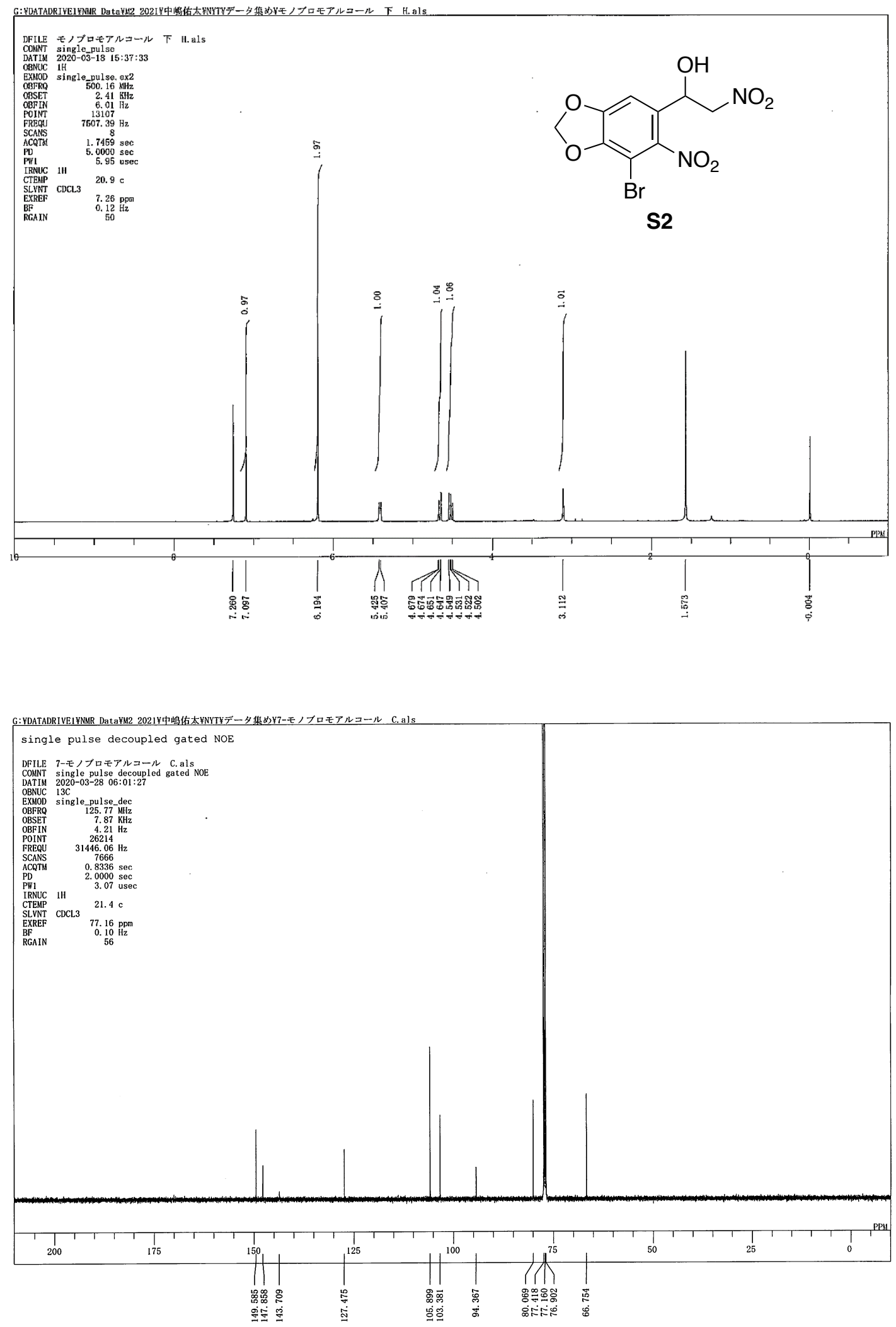

Figure S11. ${ }^{1} \mathrm{H}$ and ${ }^{13} \mathrm{C}$ NMR spectra of $\mathbf{S 2}$. 

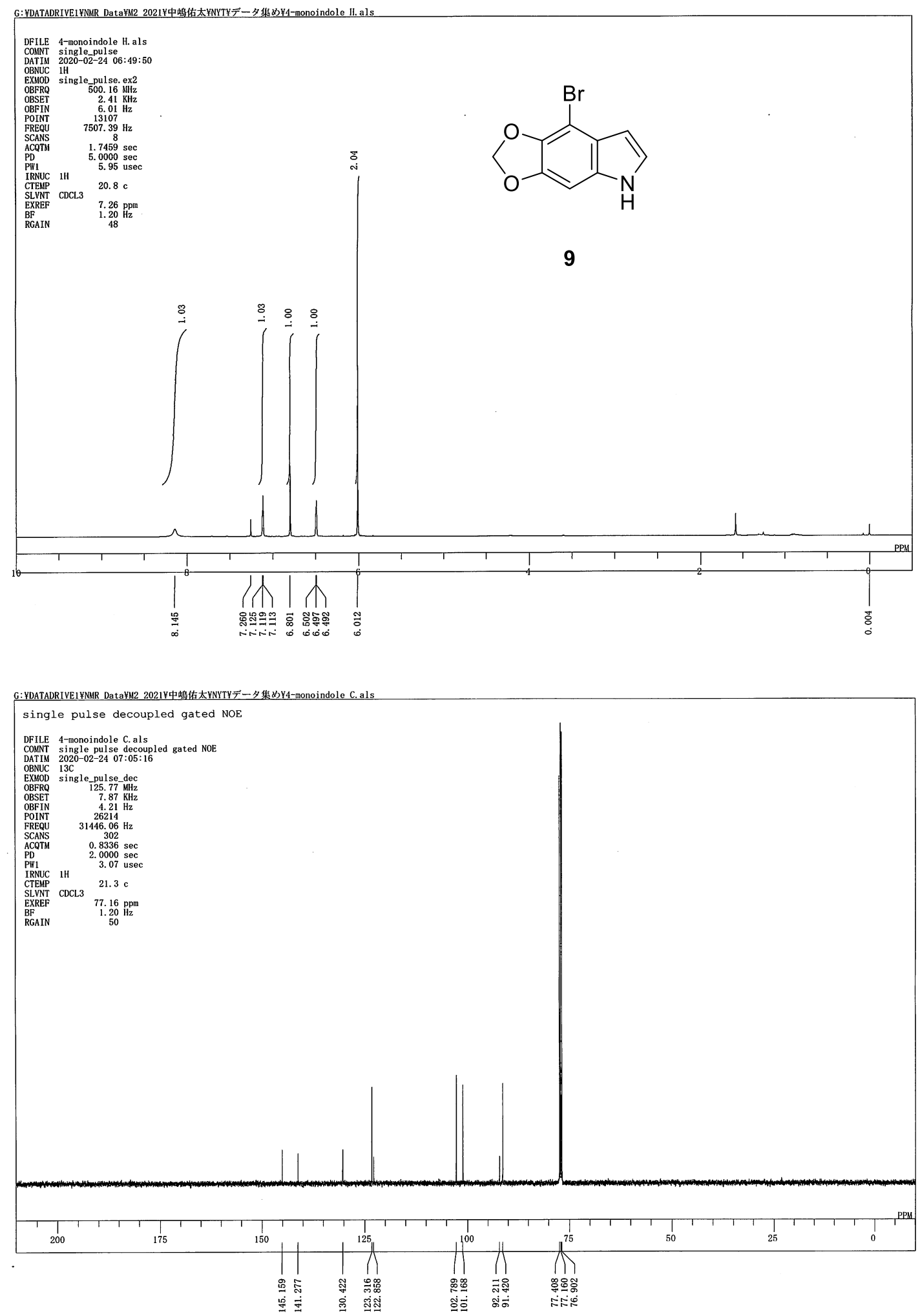

Figure S12. ${ }^{1} \mathrm{H}$ and ${ }^{13} \mathrm{C}$ NMR spectra of 9 . 

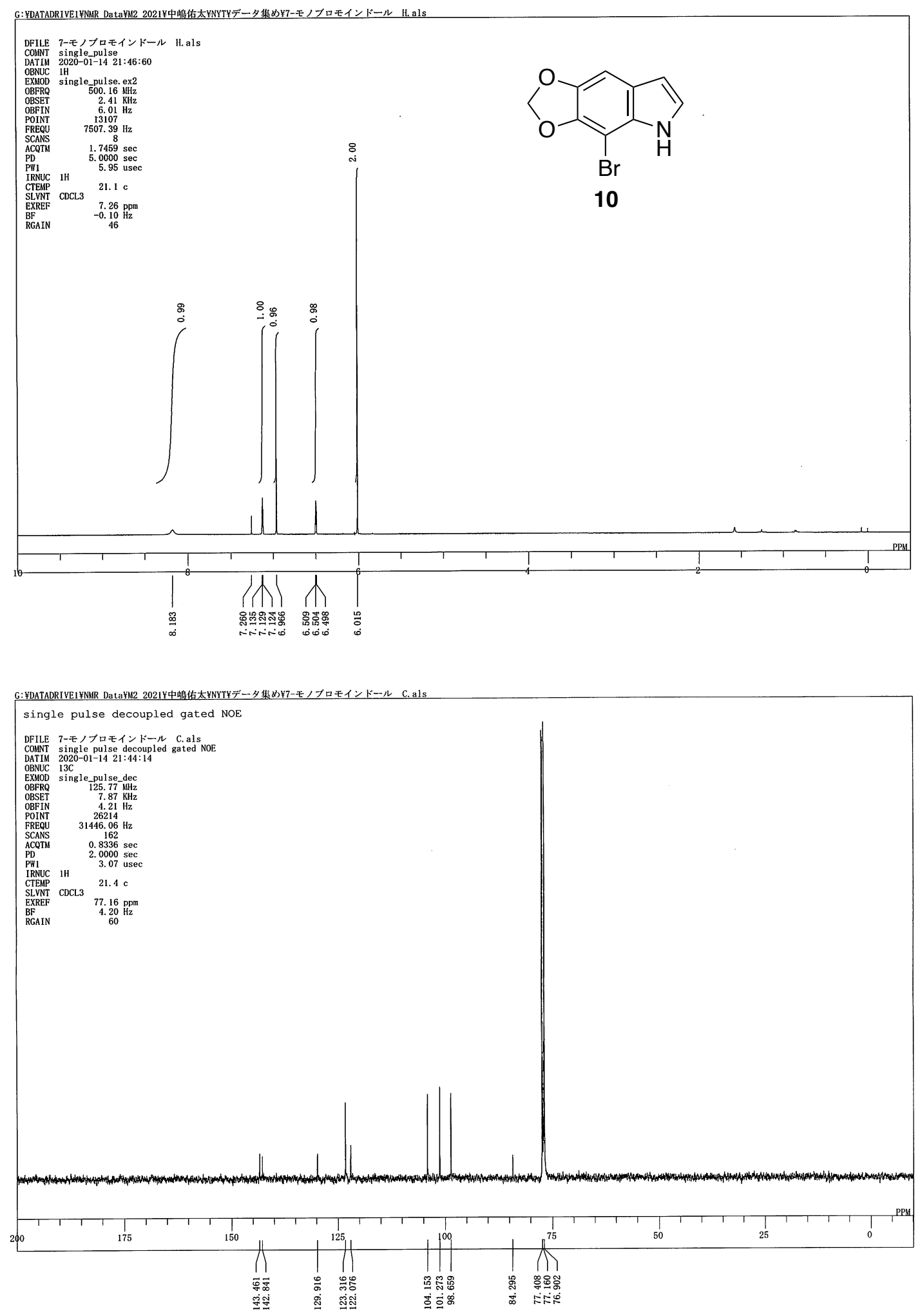

Figure S13. ${ }^{1} \mathrm{H}$ and ${ }^{13} \mathrm{C}$ NMR spectra of $\mathbf{1 0}$. 

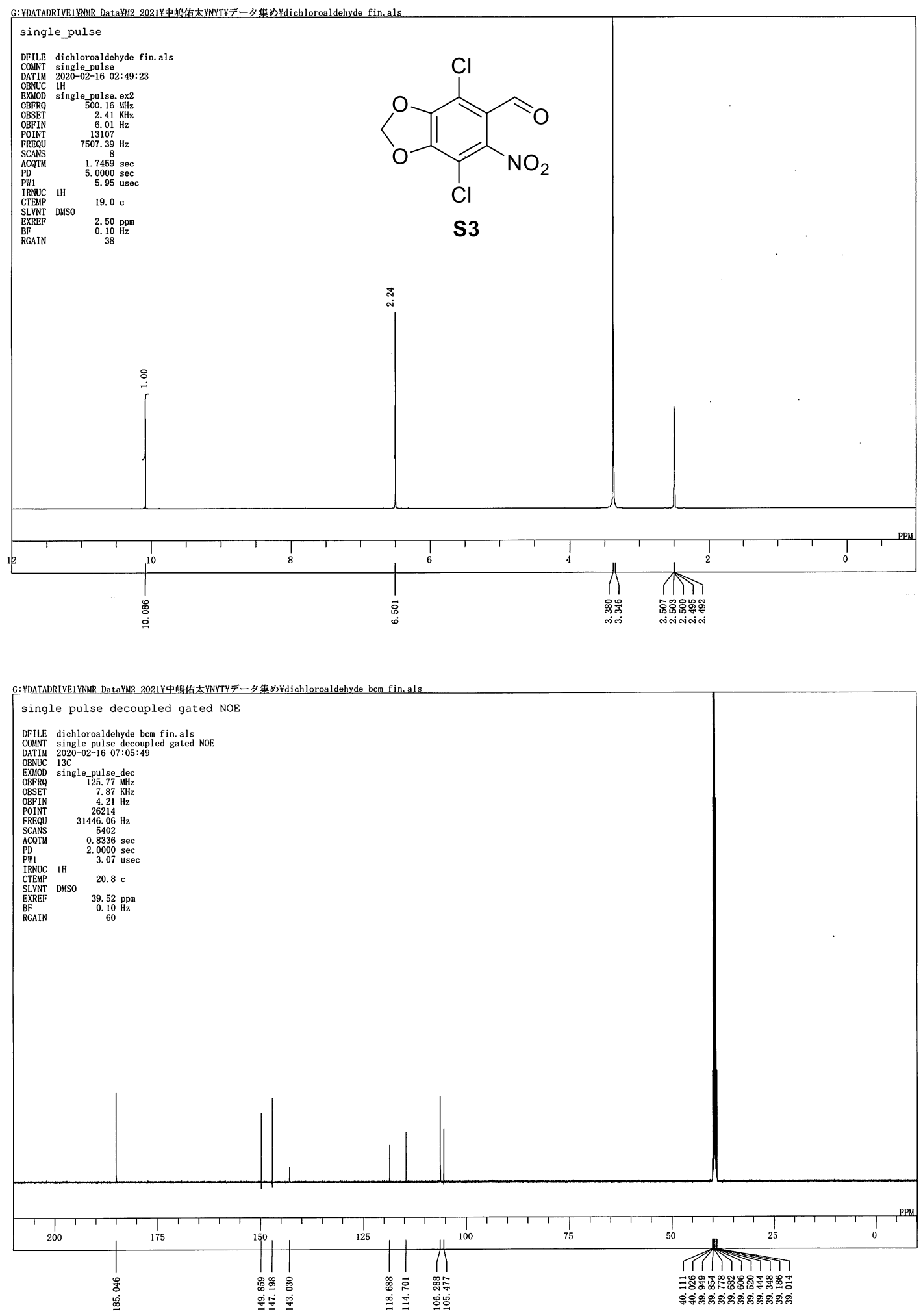

Figure S14. ${ }^{1} \mathrm{H}$ and ${ }^{13} \mathrm{C}$ NMR spectra of $\mathbf{S 3}$. 

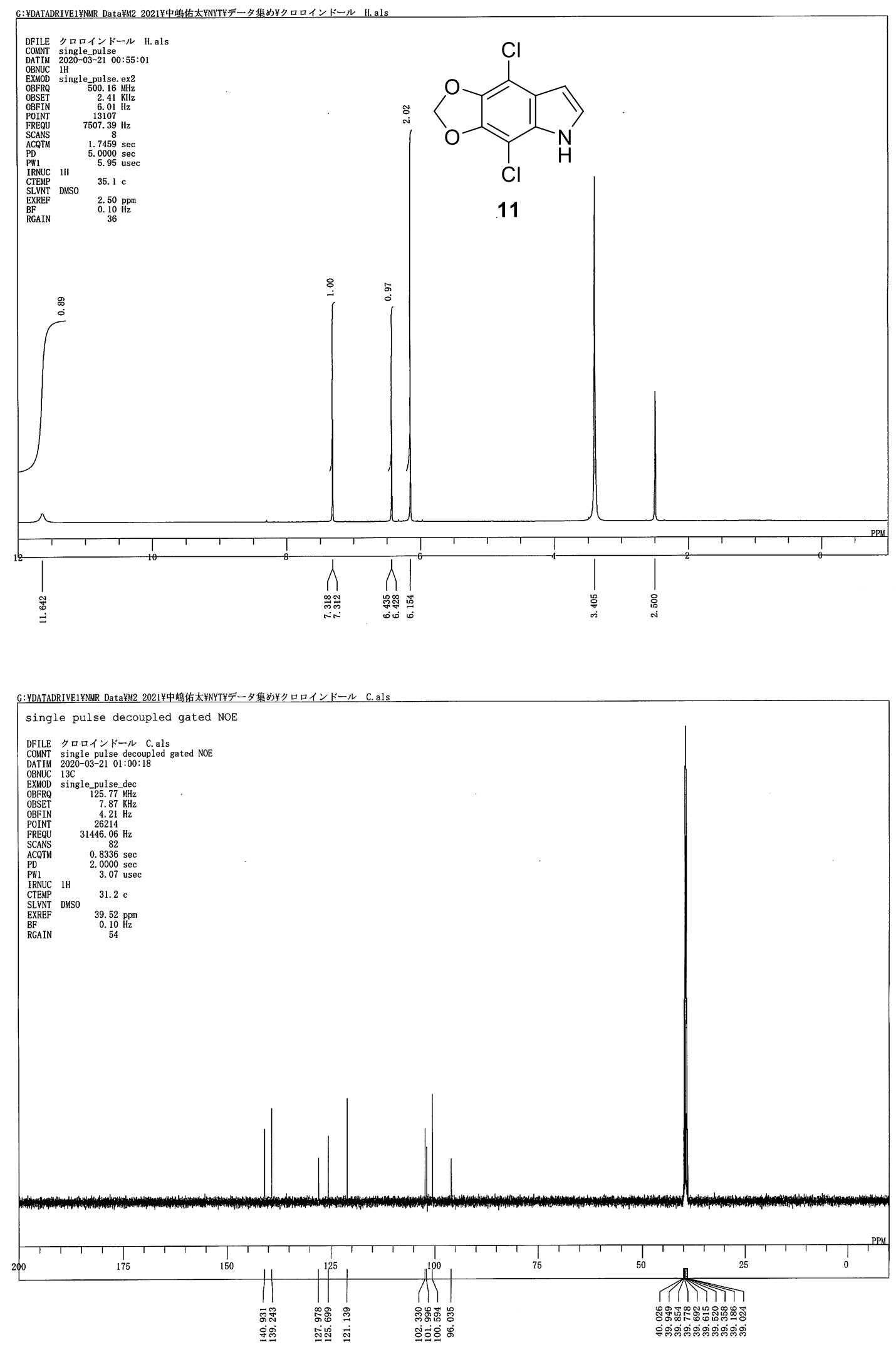

Figure S15. ${ }^{1} \mathrm{H}$ and ${ }^{13} \mathrm{C}$ NMR spectra of $\mathbf{1 1}$. 

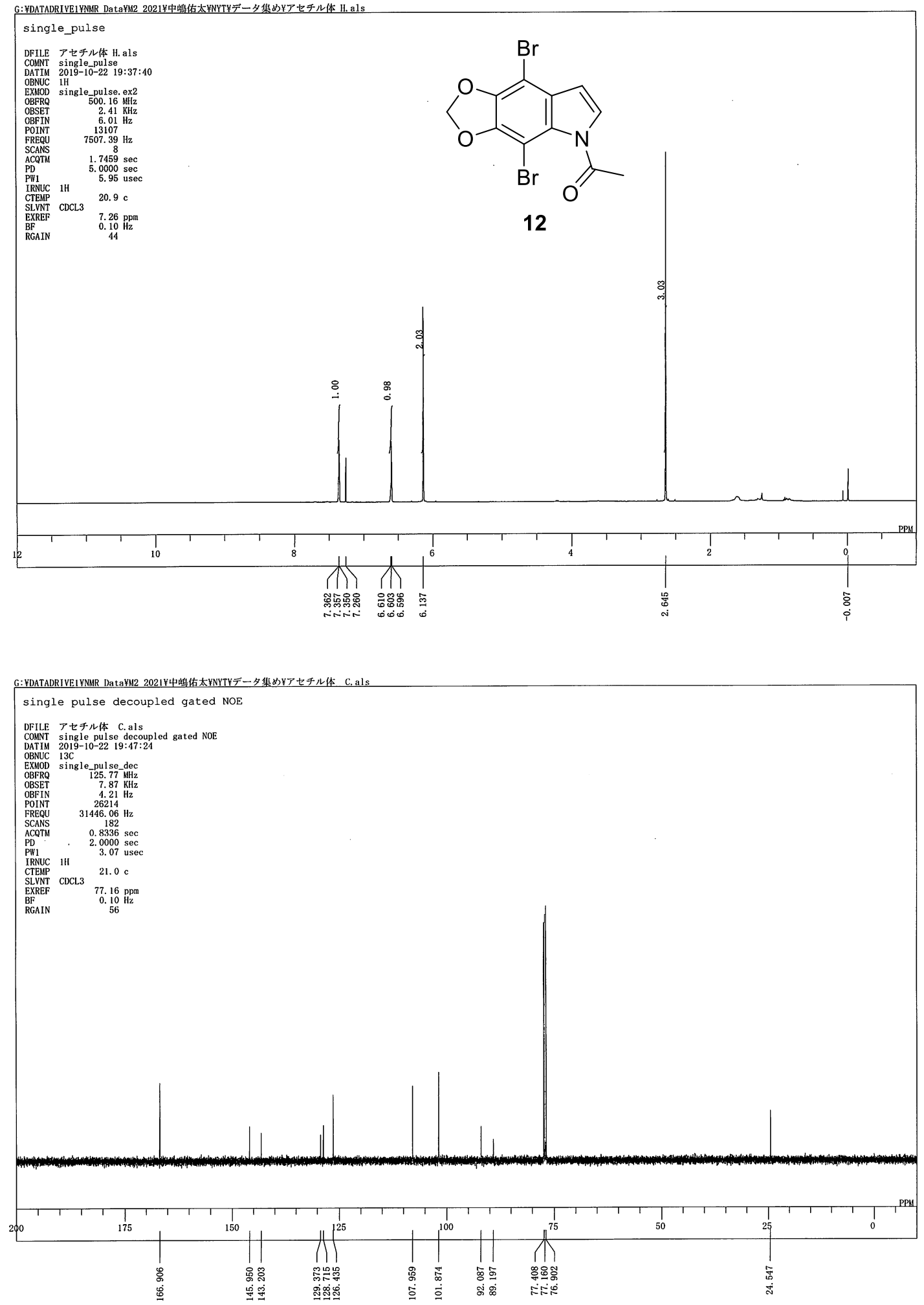

Figure S16. ${ }^{1} \mathrm{H}$ and ${ }^{13} \mathrm{C}$ NMR spectra of $\mathbf{1 2}$. 

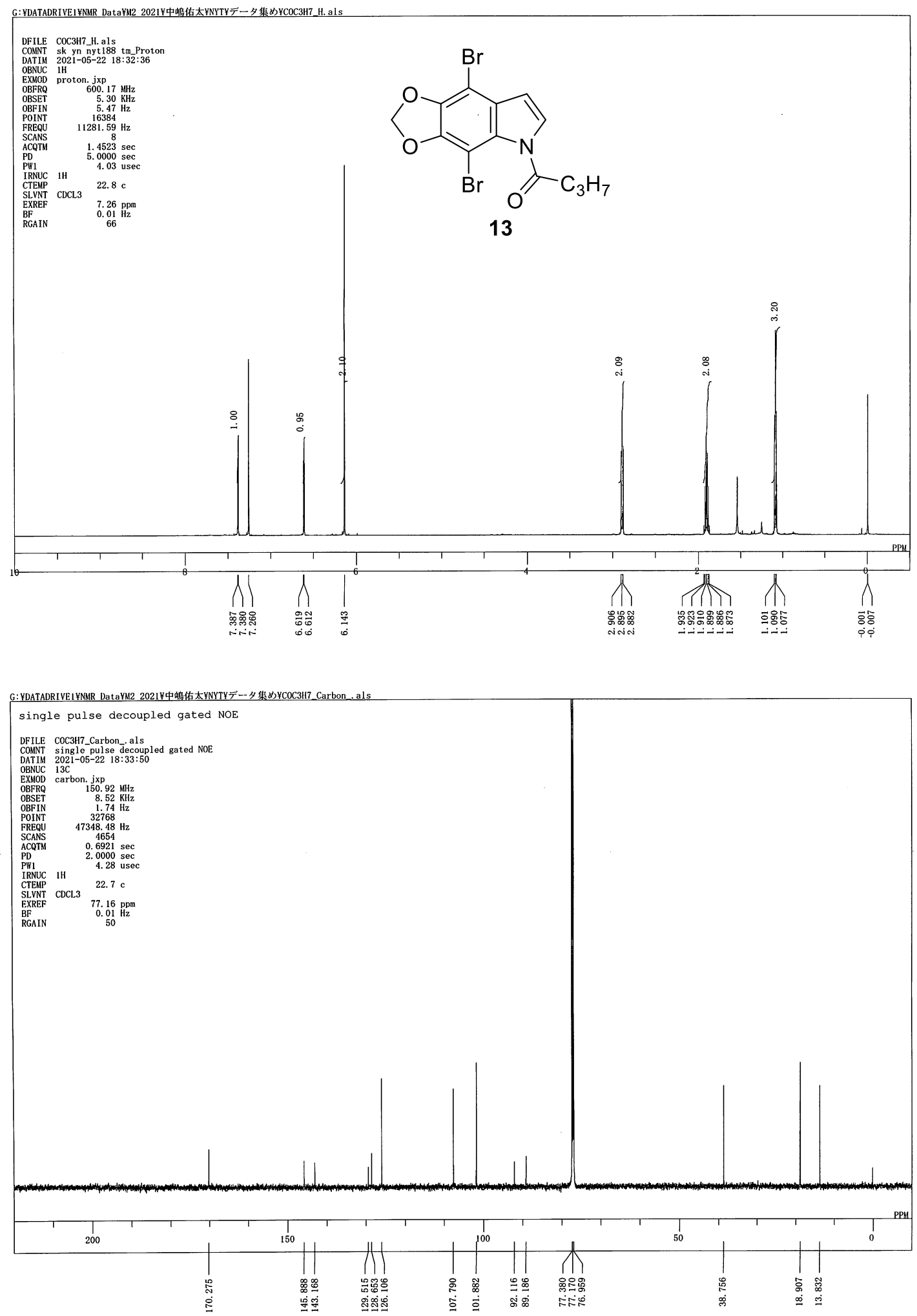

Figure S17. ${ }^{1} \mathrm{H}$ and ${ }^{13} \mathrm{C}$ NMR spectra of $\mathbf{1 3}$. 

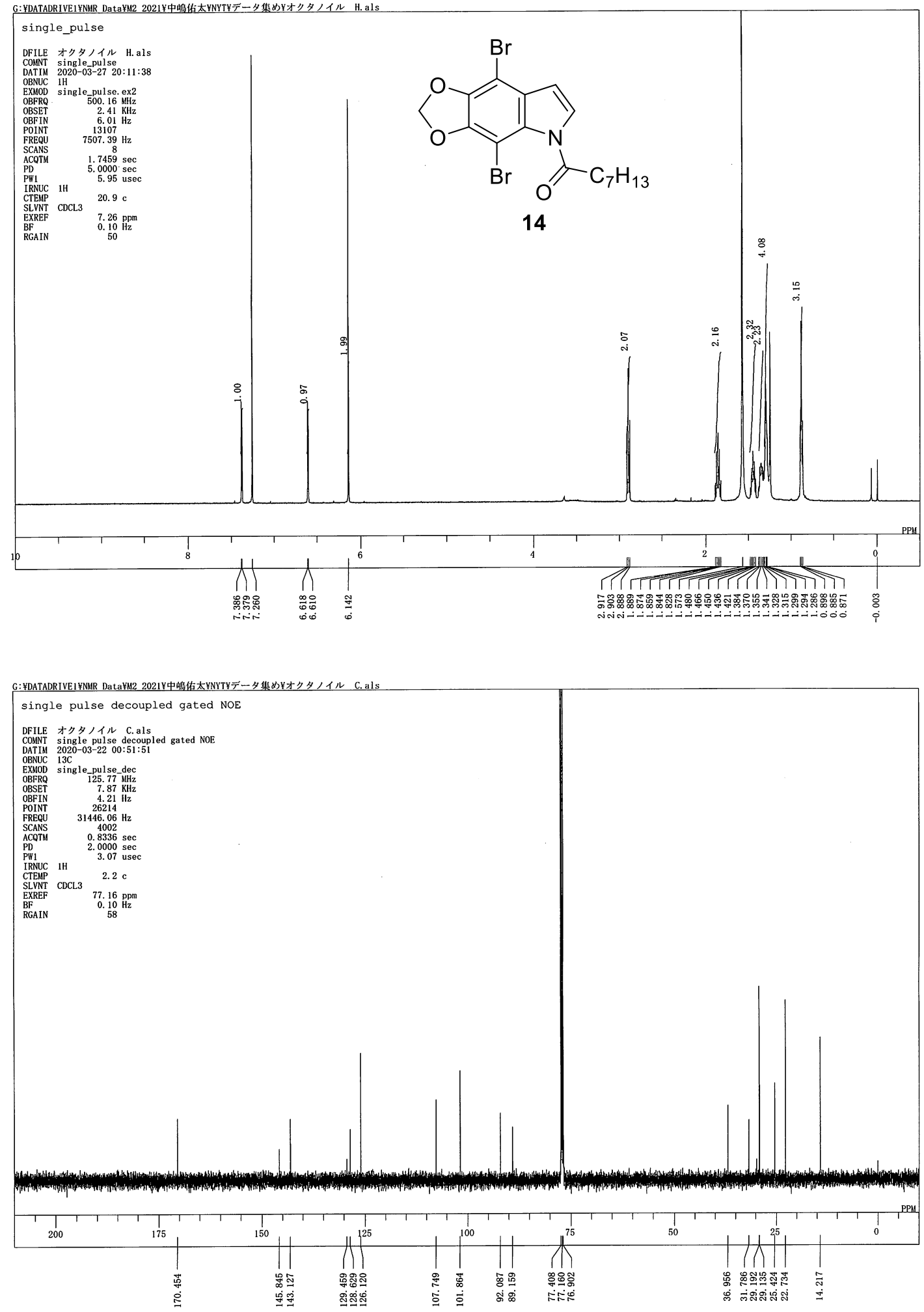

Figure S18. ${ }^{1} \mathrm{H}$ and ${ }^{13} \mathrm{C}$ NMR spectra of $\mathbf{1 4}$. 

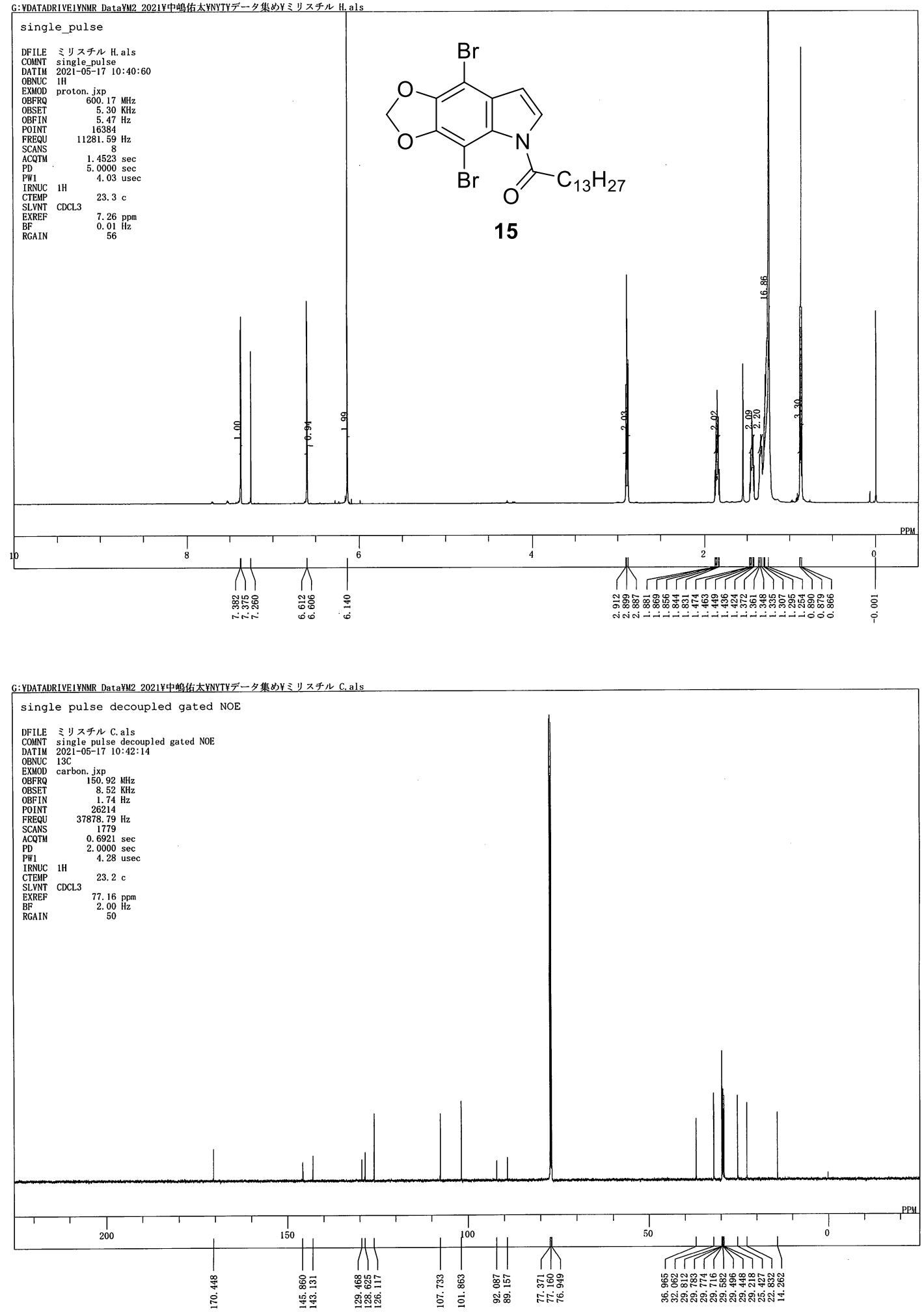

Figure S19. ${ }^{1} \mathrm{H}$ and ${ }^{13} \mathrm{C}$ NMR spectra of $\mathbf{1 5}$. 

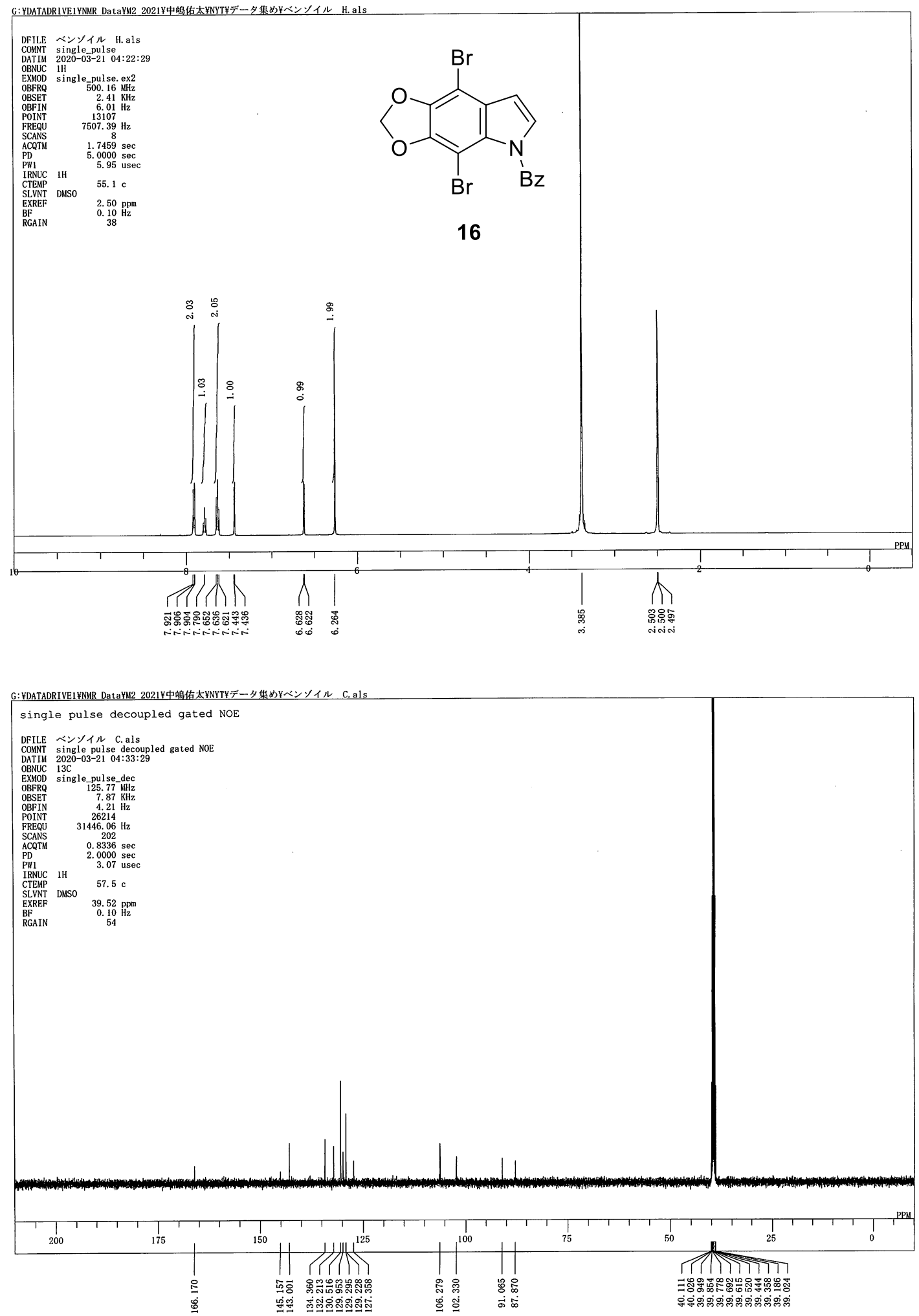

Figure S20. ${ }^{1} \mathrm{H}$ and ${ }^{13} \mathrm{C}$ NMR spectra of $\mathbf{1 6}$. 

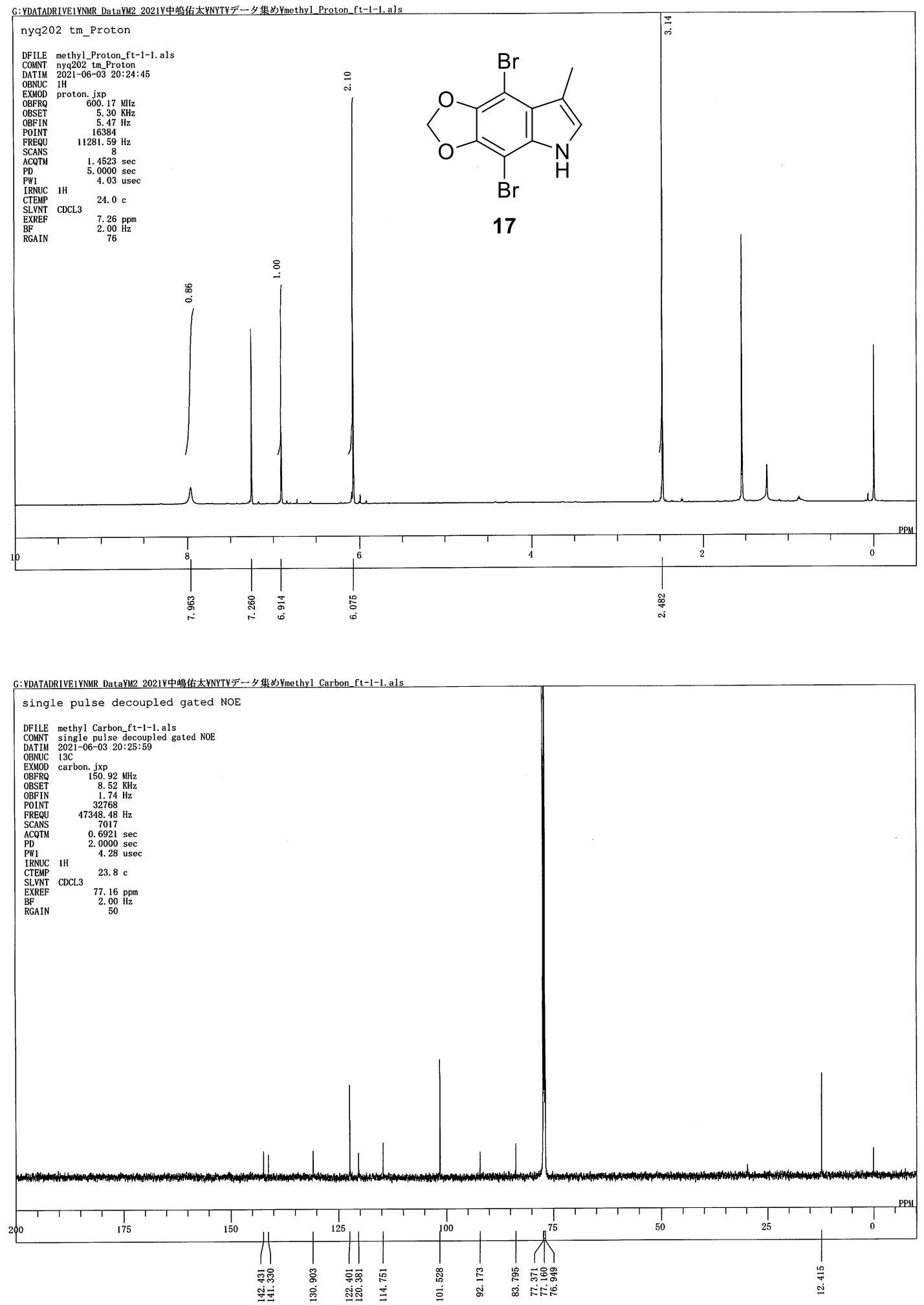

Figure S21. ${ }^{1} \mathrm{H}$ and ${ }^{13} \mathrm{C}$ NMR spectra of 17 . 

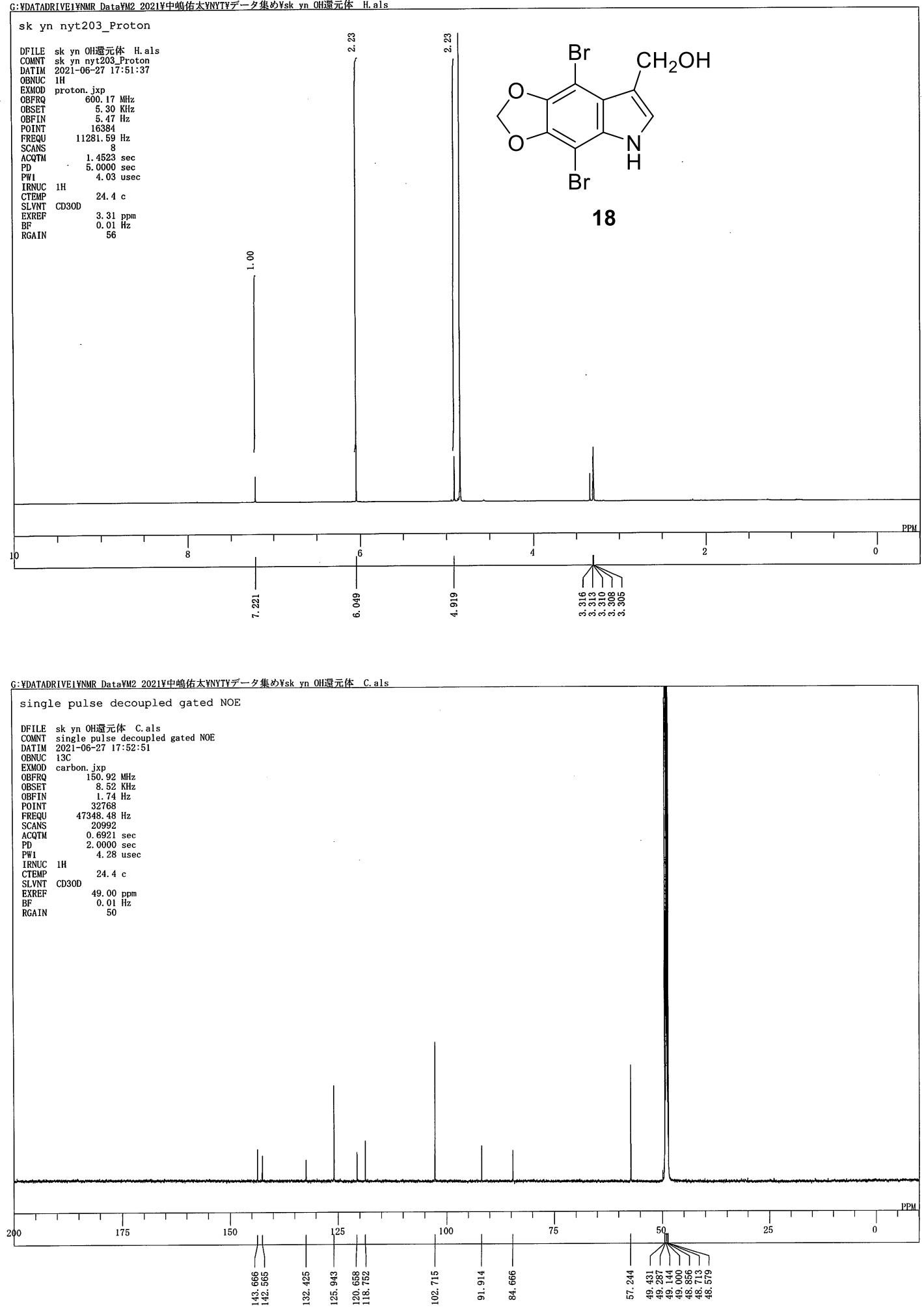

Figure S22. ${ }^{1} \mathrm{H}$ and ${ }^{13} \mathrm{C}$ NMR spectra of $\mathbf{1 8}$. 

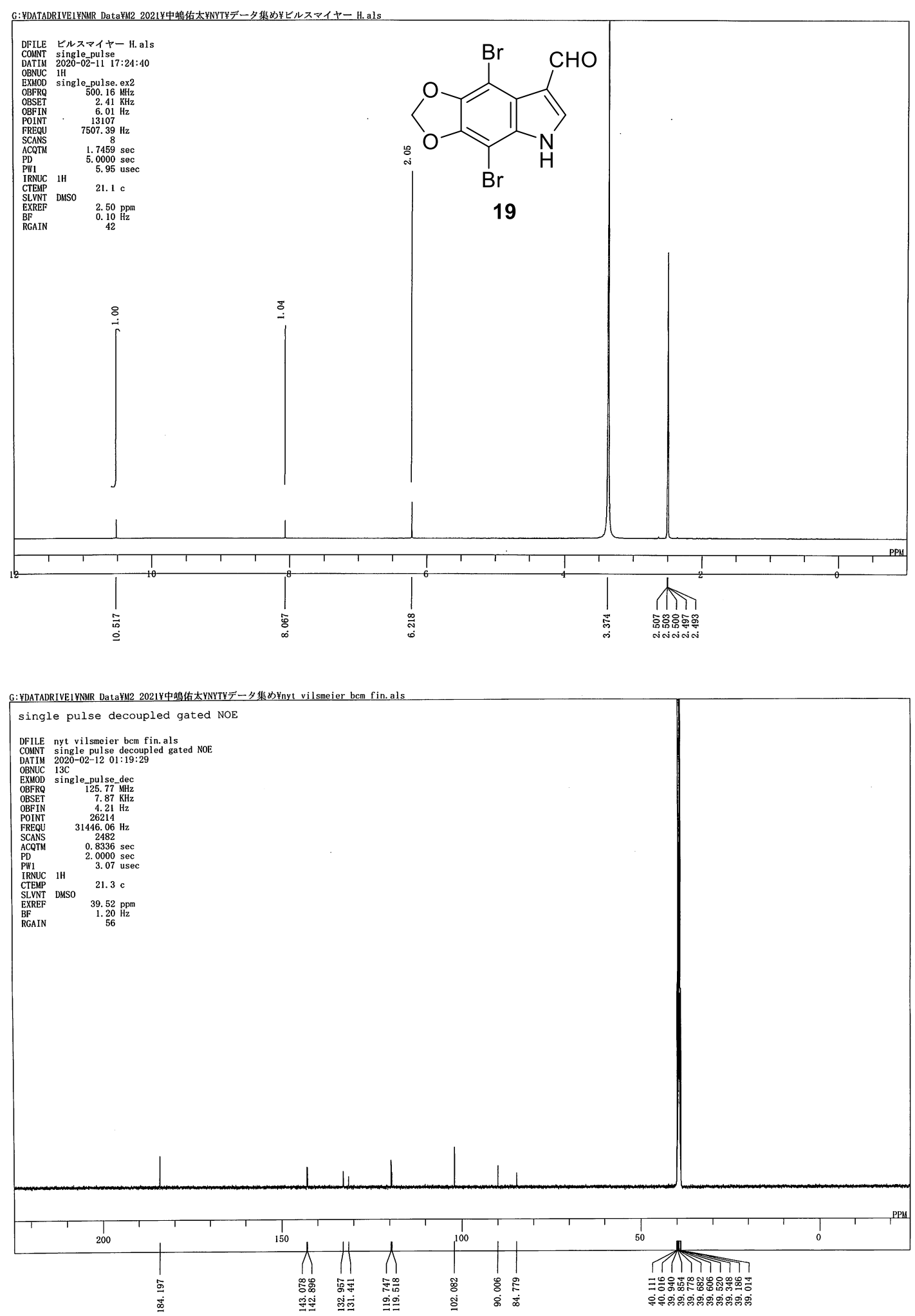

Figure S23. ${ }^{1} \mathrm{H}$ and ${ }^{13} \mathrm{C}$ NMR spectra of 19. 

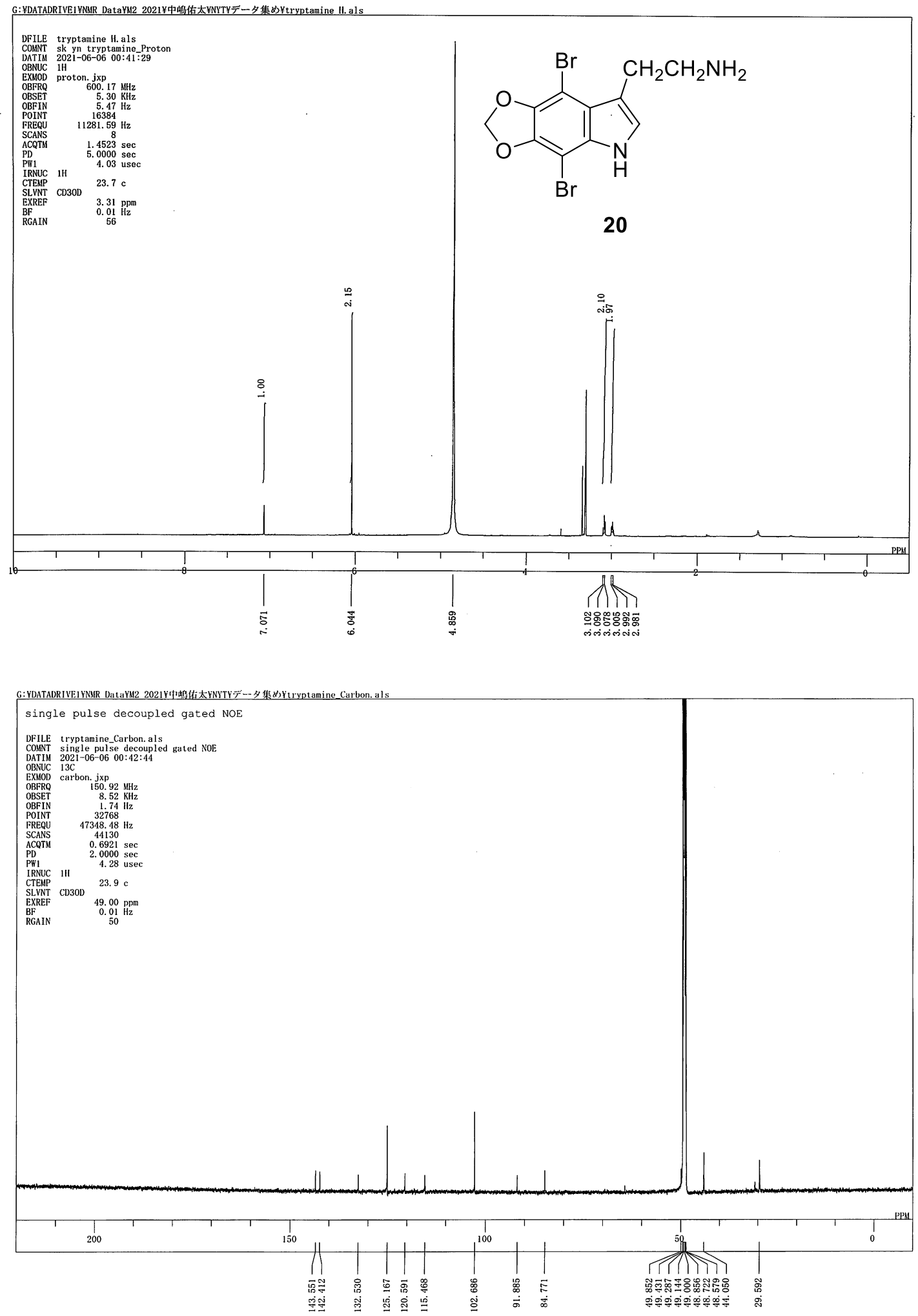

Figure S24. ${ }^{1} \mathrm{H}$ and ${ }^{13} \mathrm{C}$ NMR spectra of 20 . 
Table S1. Calculated ${ }^{13} \mathrm{C}$ NMR Data Based on $\omega \mathrm{B} 97 \mathrm{X}-\mathrm{D} / 6-31 \mathrm{G}^{*}$ for Models $\boldsymbol{a}$ and $\boldsymbol{b}$ and Comparisons with Experimental Data of $\mathbf{1}$.

\begin{tabular}{|c|c|c|c|c|c|c|c|}
\hline \multicolumn{4}{|c|}{$a$} & \multicolumn{4}{|c|}{$b$} \\
\hline No. & $\delta_{\exp }$ & $\delta_{\text {calcd }}$ & $\delta_{\text {exp }}-\delta_{\text {calcd }}$ & No. & $\delta_{\exp }$ & $\delta_{\text {calcd }}$ & $\delta_{\text {exp }}-\delta_{\text {calce }}$ \\
\hline 2 & 123.5 & 122.2 & 1.3 & 2 & 123.5 & 123.8 & -0.3 \\
\hline 3 & 103.9 & 103.7 & 0.2 & 3 & 103.9 & 104.6 & -0.7 \\
\hline 4 & 91.6 & 93.0 & -1.4 & 4 & 91.6 & 108.2 & -16.6 \\
\hline 5 & $142.7^{a}$ & 144.2 & -1.5 & 5 & 83.6 & 99.0 & -0.4 \\
\hline 6 & $141.4^{a}$ & 142.7 & -1.3 & 6 & $142.7^{a}$ & 143.1 & 11.1 \\
\hline 7 & 83.6 & 82.2 & 1.4 & 7 & $141.4^{a}$ & 130.3 & -15.4 \\
\hline 8 & 129.4 & 128.7 & 0.7 & 8 & 129.4 & 118.6 & 10.8 \\
\hline 9 & 122.9 & 123.7 & -0.8 & 9 & 122.9 & 128.5 & -5.6 \\
\hline 10 & 101.5 & 104.3 & -2.8 & 10 & 101.5 & 104.8 & -3.3 \\
\hline$\overline{\mathrm{SD}^{b}}$ & & & 1.4 & $\overline{\mathrm{SD}^{b}}$ & & & 9.1 \\
\hline
\end{tabular}<smiles>Brc1c2c(c(Br)c3[nH]ccc13)OOCO2</smiles>

a<smiles></smiles>

b 